\title{
Intramolecular Dehydro Diels-Alder Reactions of Diarylacetylenes: Switching between Benzo[b]- and Benzo[c]fluorenones as Products by Controlling the Rearrangement of Cyclic Allene Intermediates
}

David Rodríguez, María Fernanda Martínez-Esperón, Armando Navarro-Vázquez, Luis Castedo, Domingo Domínguez and Carlos Saá*

Departamento de Química Orgánica y Unidad Asociada al CSIC, Facultad de Química, Universidad de Santiago de Compostela, 15782 Santiago de Compostela, Spain

\section{Contents}

1) Experimental details and characterization data for all new compounds, pages S2S26.

2) Copies of ${ }^{1} \mathrm{H}$ NMR and ${ }^{13} \mathrm{C}$ NMR spectra for all new compounds, pages S27-S44, S45-S67, S68-S80. .

3) Cartesian coordinates and total energies for structures $\mathbf{2 a - c}, \mathbf{2 e - g}, \mathbf{2 h}-\mathbf{l}$ and $\mathbf{6 a - c}, \mathbf{6 e -}$ g, 6h-l, pages S81-S94. 


\section{Experimental Section: General details}

All reactions were carried out under argon atmosphere with magnetic stirring. The solvents were purified and dried using standard procedures. All reagents were purchased and used without further purification. ${ }^{1} \mathrm{H}$ NMR spectra at $250,13 \mathrm{MHz}$ and ${ }^{13} \mathrm{C}$ NMR spectra at 62,89 $\mathrm{MHz}$ were determined using $\mathrm{CDCl}_{3}$ as solvent with tetramethylsilane as internal standard. Mass spectra were measured by ionizing the sample at $70 \mathrm{eV}$. Column chromatographies were made on silica gel 230-400 mesh (flash).

\section{Starting materials: General procedure}

Diarylacetylenic alcohols: To a stirred solution of aryl iodide 10b-e (1.1 equiv), $\mathrm{PdCl}_{2}\left(\mathrm{PPh}_{3}\right)_{2}(0.02$ equiv) and $\mathrm{CuI}$ (0.04 equiv) in a 4:1 mixture of dry, degassed THF/Et $3 \mathrm{~N}$ at $\mathrm{rt}$ was added the starting alkyne 8 or 9 (typically, $1 \mathrm{mmol}$ ). Once starting materials have disappeared (TLC monitoring, typically $30 \mathrm{~min}$ ), the mixture was passed through a pad of Celite to remove the solids. The filtrate was concentrated and the reidue dissolved in EtOAc, washed with $5 \%$ aqueous $\mathrm{HCl}$ and brine, dried over anhydrous $\mathrm{Na}_{2} \mathrm{SO}_{4}$ and concentrated to dryness. Purification of the residue by flash chromatography on silica gel using a mixture of EtOAc/hexanes as eluent afforded the diarylacetylenic alcohol in quite good yields.

Oxidation of diarylacetylenic alcohols: To a stirred solution of the diarylacetylenic alcohol in $\mathrm{Cl}_{2} \mathrm{CH}_{2}(c=0.1-1 \mathrm{M})$ at $\mathrm{rt}$ was added $\mathrm{MnO}_{2}$ in small portions until disappearance of starting material (typically 30-60 min, TLC monitoring, EtOAc/hexanes 1:9). The mixture was passed through a pad of silica to remove the solids and concentrated (purification by chromatography gives partial desilylation). Usually, the resulting ketones $\mathbf{1 b - 0}$ and 12a-c are almost pure and no further purification is needed.

\section{1-\{2-[2-(4-methylphenyl)-1-ethynyl]phenyl\}-3-(trimethylsilyl)-2-propyn-1-ol}

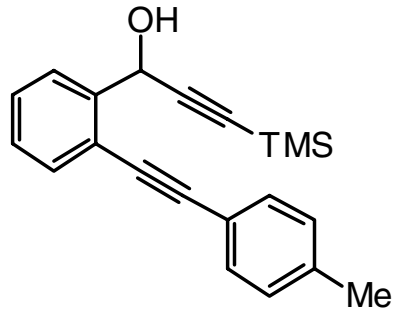

Clear oil; ${ }^{1} \mathrm{H}$ NMR $\left(\mathrm{CDCl}_{3}\right) \delta: 7.71(\mathrm{~d}, J=7.3 \mathrm{~Hz}, 1 \mathrm{H}, \mathrm{Ar} H), 7.54(\mathrm{~d}, J=7.2 \mathrm{~Hz}, 1 \mathrm{H}, \mathrm{Ar} H)$, 7.49-7.27 (m, 4H, ArH), 7.17 (d, J=7.2 Hz, 2H, ArH), 5.93 (d, J=5.5 Hz, 1H, ArCH), 2.68 $(\mathrm{d}, J=5.5 \mathrm{~Hz}, 1 \mathrm{H}, \mathrm{OH}), 2.37\left(\mathrm{~s}, 3 \mathrm{H}, \mathrm{CH}_{3}\right), 0.18\left(\mathrm{~s}, 9 \mathrm{H}, \mathrm{Si}\left(\mathrm{CH}_{3}\right)_{3}\right) ;{ }^{13} \mathrm{C} \mathrm{NMR} / \mathrm{DEPT}$

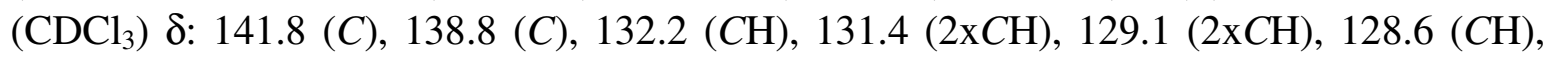
$128.2(C H), 126.7(C H), 121.7(C), 119.6(C), 104.3(C), 95.2(C), 91.3(C), 85.9(C), 63.6$ 
(CH), $21.5\left(\mathrm{CH}_{3}\right),-0.2\left(\mathrm{Si}\left(\mathrm{CH}_{3}\right)_{3}\right) ; \mathrm{MS}(70 \mathrm{eV}) \mathrm{m} / \mathrm{z}(\%): 318\left(\mathrm{M}^{+}, 7\right), 303(19), 229(19), 73$ $(100)$.

1-\{2-[2-(4-methylphenyl)-1-ethynyl]phenyl\}-3-(trimethylsilyl)propynone $\mathbf{1 b}$

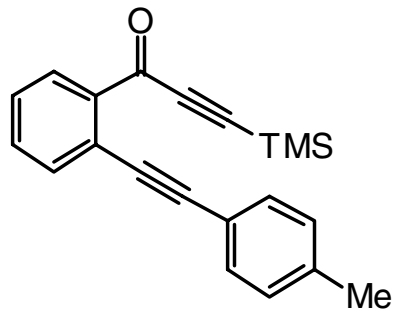

Clear oil; ${ }^{1} \mathrm{H}$ NMR $\left(\mathrm{CDCl}_{3}\right) \delta: 8.13(\mathrm{~d}, J=8.9 \mathrm{~Hz}, 1 \mathrm{H}, \mathrm{Ar} H), 7.63(\mathrm{~d}, J=7.5 \mathrm{~Hz}, 1 \mathrm{H}, \operatorname{Ar} H)$, 7.57-7.37 (m, 4H, $\mathrm{ArH}), 7.16(\mathrm{~d}, J=9.5 \mathrm{~Hz}, 2 \mathrm{H}, \mathrm{ArH}), 2.37$ (s, 3H, $\left.\mathrm{CH}_{3}\right), 0.25$ (s, 9H, $\left.\mathrm{Si}\left(\mathrm{CH}_{3}\right)_{3}\right) ;{ }^{13} \mathrm{C}$ NMR/DEPT $\left(\mathrm{CDCl}_{3}\right)$ \&: $177.0(\mathrm{CO}), 138.8(\mathrm{C}), 137.6(\mathrm{C}), 134.0(\mathrm{CH})$, $132.5(\mathrm{CH}), 132.0(\mathrm{CH}), 131.8(2 \times C H), 129.0(2 \times C H), 127.6(\mathrm{CH}), 123.1(C), 120.1(C)$, 101.6 (C), $100.3(C), 95.6(C), 87.6(C), 21.5\left(\mathrm{CH}_{3}\right),-0.8\left(\mathrm{Si}\left(\mathrm{CH}_{3}\right)_{3}\right)$; $\mathrm{MS}(70 \mathrm{eV}) \mathrm{m} / z(\%)$ : $316\left(\mathrm{M}^{+}, 67\right), 301(100), 273(54), 271(31), 189(22)$.

1-\{2-[2-(4-methoxyphenyl)-1-ethynyl]phenyl\}-3-(trimethylsilyl)-2-propyn-1-ol

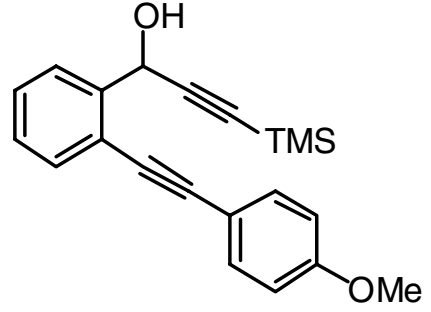

Clear oil; ${ }^{1} \mathrm{H}$ NMR $\left(\mathrm{CDCl}_{3}\right) \delta: 7.71(\mathrm{dd}, J=7.6,1.5 \mathrm{~Hz}, 1 \mathrm{H}, \mathrm{Ar} H), 7.56-7.45(\mathrm{~m}, 3 \mathrm{H}, \mathrm{Ar} H)$, 7.41-7.27 (m, 2H, $\operatorname{Ar} H$ ), 6.93-6.85 (m, 2H, $\operatorname{Ar} H), 5.93$ (broad s, $1 \mathrm{H}, \operatorname{ArCH}), 3.83$ (s, 3H, $\left.\mathrm{OCH}_{3}\right), 2.80($ broad s, $1 \mathrm{H}, \mathrm{OH}), 0.18\left(\mathrm{~s}, 9 \mathrm{H}, \mathrm{Si}\left(\mathrm{CH}_{3}\right)_{3}\right) ;{ }^{13} \mathrm{C}$ NMR/DEPT $\left(\mathrm{CDCl}_{3}\right) \delta: 159.8$ (C), $141.7(C), 133.0(2 \times C H), 132.1(\mathrm{CH}), 128.5(\mathrm{CH}), 128.2(\mathrm{CH}), 126.7(\mathrm{CH}), 121.9(\mathrm{C})$, 114.8 (C), 114.0 (2xCH), 104.3 (C), $95.1(C), 91.4(C), 85.3(C), 63.7(C H), 55.3\left(C_{3}\right)$, $0.2\left(\mathrm{Si}\left(\mathrm{CH}_{3}\right)_{3}\right) ; \mathrm{MS}(70 \mathrm{eV}) \mathrm{m} / \mathrm{z}(\%): 334\left(\mathrm{M}^{+}, 5\right), 319(25), 108(85), 73(100)$.

1-\{2-[2-(4-methoxyphenyl)-1-ethynyl]phenyl\}-3-(trimethylsilyl)propynone 1c

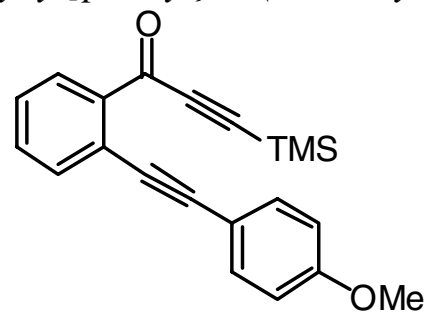

Clear oil; ${ }^{1} \mathrm{H}$ NMR $\left(\mathrm{CDCl}_{3}\right) \delta: 8.13(\mathrm{dd}, J=7.7,1.3 \mathrm{~Hz}, 1 \mathrm{H}, \mathrm{Ar} H), 7.64-7.48(\mathrm{~m}, 4 \mathrm{H}, \mathrm{Ar} H)$, $7.41(\mathrm{td}, J=7.5,1.6 \mathrm{~Hz}, 1 \mathrm{H}, \mathrm{ArH}), 6.91-6.87(\mathrm{~m}, 2 \mathrm{H}, \mathrm{Ar} H), 3.83$ (s, 3H, OCH $\mathrm{OCH}_{3}, 0.25$ (s, 9H, $\left.\mathrm{Si}\left(\mathrm{CH}_{3}\right)_{3}\right) ;{ }^{13} \mathrm{C}$ NMR/DEPT $\left(\mathrm{CDCl}_{3}\right) \delta: 177.1(\mathrm{CO}), 159.9(\mathrm{C}), 137.4(\mathrm{C}), 133.9(\mathrm{CH})$, $133.5(2 \times C H), 132.5(\mathrm{CH}), 132.1(\mathrm{CH}), 127.4(\mathrm{CH}), 123.8(\mathrm{C}), 115.3(\mathrm{C}), 113.9(2 \times C \mathrm{H})$, 101.6 (C), $100.4(C), 95.8(C), 87.1(C), 55.2\left(\mathrm{CH}_{3}\right),-0.8\left(\mathrm{Si}\left(\mathrm{CH}_{3}\right)_{3}\right)$; $\mathrm{MS}(70 \mathrm{eV}) \mathrm{m} / z(\%)$ : $332\left(\mathrm{M}^{+}, 100\right), 317$ (71), $289(30), 274(47)$.

4-\{2-[1-Hydroxy-3-(trimethylsilyl)-2-propynyl]-phenylethynyl\}-phenol 


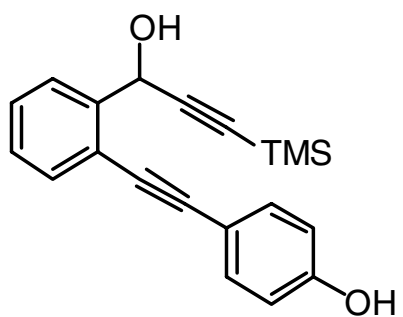

Clear oil; ${ }^{1} \mathrm{H}$ NMR $\left(\mathrm{CDCl}_{3}\right) \delta: 7.74(\mathrm{~d}, J=7.5 \mathrm{~Hz}, 1 \mathrm{H}, \mathrm{Ar} H), 7.64$ (broad s, $\left.1 \mathrm{H}, \mathrm{ArH}\right), 7.52$ $(\mathrm{d}, J=7.2 \mathrm{~Hz}, 1 \mathrm{H}, \operatorname{Ar} H), 7.40-7.30(\mathrm{~m}, 4 \mathrm{H}, \operatorname{Ar} H), 6.83(\mathrm{~d}, J=7.2 \mathrm{~Hz}, 1 \mathrm{H}, \operatorname{Ar} H), 6.03$ (s, $1 \mathrm{H}, \mathrm{ArCH}), 3.79$ (broad s, 1H, OH), $0.20\left(\mathrm{~s}, 9 \mathrm{H}, \mathrm{Si}\left(\mathrm{CH}_{3}\right)_{3}\right) ;{ }^{13} \mathrm{C}$ NMR/DEPT $\left(\mathrm{CDCl}_{3}\right) \delta$ : $156.6(\mathrm{C}), 141.0(\mathrm{C}), 133.0(2 \times \mathrm{CH}), 132.0(\mathrm{CH}), 128.3(\mathrm{CH}), 128.2(\mathrm{CH}), 126.7(\mathrm{CH})$, 121.9 (C), 115.6 (2 x CH), 114.1 (C), 104.0 (C), $95.3(C), 91.7(C), 84.8(C), 63.6(C H),-$ $0.3\left(\mathrm{Si}\left(\mathrm{CH}_{3}\right)_{3}\right)$; MS (70 eV) m/z (\%): $320\left(\mathrm{M}^{+}, 38\right), 319(25), 305$ (90), 231 (90), $226(62)$, 211 (100); HRMS $\left(\mathrm{C}_{20} \mathrm{H}_{20} \mathrm{O}_{2} \mathrm{Si}\right)$ : calc. 320.1232; found, 320.1225 .

1-\{2-[2-(4-hydroxyphenyl)-1-ethynyl]phenyl\}-3-(trimethylsilyl)propynone $\mathbf{1 d}$

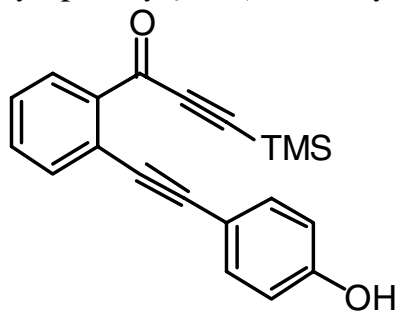

Clear oil; ${ }^{1} \mathrm{H}$ NMR $\left(\mathrm{CDCl}_{3}\right) \delta: 8.13(\mathrm{~d}, J=9.1 \mathrm{~Hz}, 1 \mathrm{H}, \mathrm{Ar} H), 7.71-7.33(\mathrm{~m}, 6 \mathrm{H}, \mathrm{Ar} H), 6.81$ $(\mathrm{d}, J=9.1 \mathrm{~Hz}, 1 \mathrm{H}, \mathrm{ArH}), 0.26\left(\mathrm{~s}, 9 \mathrm{H}, \mathrm{Si}\left(\mathrm{CH}_{3}\right)_{3}\right) ;{ }^{13} \mathrm{C}$ NMR/DEPT $\left(\mathrm{CDCl}_{3}\right) \delta: 177.9(\mathrm{CO})$, $156.9(\mathrm{C}), 137.0(\mathrm{C}), 134.0(\mathrm{CH}), 133.6(2 \times \mathrm{CH}), 132.8(\mathrm{CH}), 132.4(\mathrm{CH}), 127.4(\mathrm{CH})$, $123.6(C), 115.8(2 \times C H), 114.7(C), 101.6(C), 101.5(C), 96.1(C), 86.8(C),-0.8$ $\left(\mathrm{Si}\left(\mathrm{CH}_{3}\right)_{3}\right) ; \mathrm{MS}(70 \mathrm{eV}) \mathrm{m} / z(\%): 318\left(\mathrm{M}^{+}, 100\right), 303$ (92), 275 (40); HRMS $\left(\mathrm{C}_{20} \mathrm{H}_{18} \mathrm{O}_{2} \mathrm{Si}\right)$ : calc. 318.1076; found, 318.1070.

1-\{2-[2-(4-nitrophenyl)-1-ethynyl]phenyl\}-3-(trimethylsilyl)-2-propyn-1-ol<smiles>CC#CC(O)c1ccccc1C#Cc1ccc([N+](=O)[O-])cc1</smiles>

Clear oil; ${ }^{1} \mathrm{H}$ NMR $\left(\mathrm{CDCl}_{3}\right) \delta: 8.22(\mathrm{~d}, J=9.0 \mathrm{~Hz}, 2 \mathrm{H}, \operatorname{Ar} H), 7.75(\mathrm{~d}, J=7.0 \mathrm{~Hz}, 1 \mathrm{H}, \operatorname{Ar} H)$, $7.69(\mathrm{~d}, J=9.0 \mathrm{~Hz}, 2 \mathrm{H}, \operatorname{Ar} H), 7.58(\mathrm{~d}, J=7.0 \mathrm{~Hz}, 1 \mathrm{H}, \operatorname{Ar} H), 7.45(\mathrm{t}, J=7.0 \mathrm{~Hz}, 1 \mathrm{H}, \operatorname{Ar} H)$, 7.39 (t, $J=7.0 \mathrm{~Hz}, 1 \mathrm{H}, \mathrm{ArH}$ ), 5.90 (broad s, $1 \mathrm{H}, \mathrm{ArCH}$ ), 2.52 (broad s, $1 \mathrm{H}, \mathrm{OH}$ ), 0.16 (s, 9H, Si $\left.\left(\mathrm{CH}_{3}\right)_{3}\right) ;{ }^{13} \mathrm{C}$ NMR/DEPT $\left(\mathrm{CDCl}_{3}\right) \delta: 146.9(\mathrm{C}), 142.3(\mathrm{C}), 132.5(\mathrm{CH}), 132.1$ (2xCH), $129.7(\mathrm{CH}), 129.7(C), 128.2(\mathrm{CH}), 126.6(\mathrm{CH}), 123.4(2 \times C H), 120.3(C), 104.3$ (C), $92.7(C), 91.8(C), 91.4(C), 63.1(\mathrm{CH}),-0.3\left(\mathrm{Si}\left(\mathrm{CH}_{3}\right)_{3}\right)$; $\mathrm{MS}(70 \mathrm{eV}) \mathrm{m} / z(\%): 349\left(\mathrm{M}^{+}\right.$, 3), 334 (14), 288 (11), 202 (15), 73 (100).

1-\{2-[2-(4-nitrophenyl)-1-ethynyl]phenyl\}-3-(trimethylsilyl)propynone $\mathbf{1 e}$ 


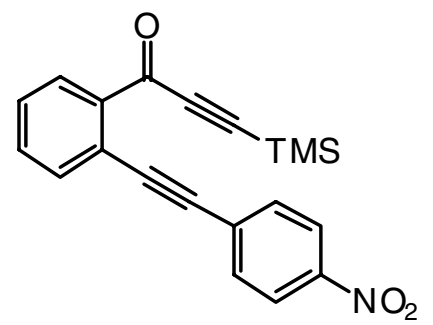

Pale brown powder; m.p. 84-85 ${ }^{\circ} \mathrm{C}$ (EtOAc); ${ }^{1} \mathrm{H}$ NMR $\left(\mathrm{CDCl}_{3}\right)$ 8: 8.26-8.18 (m, 3H, $\left.\mathrm{ArH}\right)$, 7.79-7.47 (m, 5H, $\mathrm{ArH}), 0.29$ (s, 9H, $\left.\mathrm{Si}\left(\mathrm{CH}_{3}\right)_{3}\right) ;{ }^{13} \mathrm{C} \mathrm{NMR/DEPT}\left(\mathrm{CDCl}_{3}\right) \delta: 176.6(\mathrm{CO})$, $147.0(C), 137.7(C), 134.4(\mathrm{CH}), 132.7(\mathrm{CH}), 132.6(\mathrm{CH}), 132.5(2 \times C H), 130.1(C), 128.9$ $(\mathrm{CH}), 123.5(2 \times C \mathrm{H}), 121.5(\mathrm{C}), 101.2(\mathrm{C}), 100.8(\mathrm{C}), 93.3(\mathrm{C}), 92.5(\mathrm{C}),-0.8\left(\mathrm{Si}\left(\mathrm{CH}_{3}\right)_{3}\right)$; MS (70 eV) m/z (\%): $347\left(\mathrm{M}^{+}, 52\right), 332$ (50), 287 (100), 215 (55), 163 (57), 58 (83).

1-\{2-[2-(3-methylphenyl)-1-ethynyl]phenyl\}-3-(trimethylsilyl)-2-propyn-1-ol<smiles>CC#CC(O)c1ccccc1C#Cc1cccc(C)c1</smiles>

Clear oil; ${ }^{1} \mathrm{H}$ NMR $\left(\mathrm{CDCl}_{3}\right) \delta: 7.78(\mathrm{~d}, J=7.5 \mathrm{~Hz}, 1 \mathrm{H}, \mathrm{Ar} H), 7.60(\mathrm{~d}, J=7.5 \mathrm{~Hz}, 1 \mathrm{H}, \operatorname{Ar} H)$, 7.50-7.18 (m, 6H, ArH), 6.00 (broad s, 1H, ArCH), 2.42 (broad s, $1 \mathrm{H}, \mathrm{OH}), 2.42(\mathrm{~s}, 3 \mathrm{H}$, $\left.\mathrm{CH}_{3}\right), 0.24$ (s, 9H, $\left.\mathrm{Si}\left(\mathrm{CH}_{3}\right)_{3}\right) ;{ }^{13} \mathrm{C}$ NMR/DEPT $\left(\mathrm{CDCl}_{3}\right) \delta: 141.9(\mathrm{C}), 138.0(\mathrm{C}), 132.3$ $(\mathrm{CH}), 132.1(\mathrm{CH}), 129.5(\mathrm{CH}), 128.8(\mathrm{CH}), 128.6(\mathrm{CH}), 128.3(2 \mathrm{xCH}), 126.7(\mathrm{CH}), 122.5$ (C), $121.6(C), 104.3(C), 95.2(C), 91.4(C), 86.1(C), 63.7(C H), 21.2\left(C_{3}\right),-0.2$ $\left(\mathrm{Si}\left(\mathrm{CH}_{3}\right)_{3}\right) ; \mathrm{MS}(70 \mathrm{eV}) \mathrm{m} / z(\%): 318\left(\mathrm{M}^{+}, 6\right), 303(22), 229(21), 73(100)$. 1-\{2-[2-(3-methylphenyl)-1-ethynyl]phenyl\}-3-(trimethylsilyl)propynone $\mathbf{1 f}$<smiles>CC#CC(=O)c1ccccc1C#Cc1cccc(C)c1</smiles>

Clear oil; ${ }^{1} \mathrm{H}$ NMR $\left(\mathrm{CDCl}_{3}\right) \delta: 8.13(\mathrm{~d}, J=7.0 \mathrm{~Hz}, 1 \mathrm{H}, \mathrm{Ar} H), 7.64(\mathrm{~d}, J=7.5 \mathrm{~Hz}, 1 \mathrm{H}, \mathrm{Ar} H)$, 7.57-7.37 (m, 4H, $\mathrm{ArH}), 7.29-7.12(\mathrm{~m}, 2 \mathrm{H}, \mathrm{ArH}), 2.35$ (s, 3H, $\left.\mathrm{CH}_{3}\right), 0.25$ (s, 9H, Si $\left.\left(\mathrm{CH}_{3}\right)_{3}\right)$; ${ }^{13} \mathrm{C} \mathrm{NMR/DEPT}\left(\mathrm{CDCl}_{3}\right) \delta: 177.1(\mathrm{CO}), 137.9(\mathrm{C}), 137.7(\mathrm{C}), 134.1(\mathrm{CH}), 132.5(2 \mathrm{x} C \mathrm{H})$, 132.0 $(\mathrm{CH}), 129.5(\mathrm{CH}), 129.0(\mathrm{CH}), 128.2(\mathrm{CH}), 127.7(\mathrm{CH}), 123.0(\mathrm{C}), 122.9(\mathrm{C}), 101.6$ (C), $100.4(C), 95.5(C), 87.8(C), 21.2\left(\mathrm{CH}_{3}\right),-0.8\left(\mathrm{Si}\left(\mathrm{CH}_{3}\right)_{3}\right)$; $\mathrm{MS}(70 \mathrm{eV}) \mathrm{m} / z(\%): 316$ $\left(\mathrm{M}^{+}, 42\right), 302$ (27), 301 (95), 273 (57), 189 (55), 73 (55), 58 (100).

1-\{2-[2-(3-methoxyphenyl)-1-ethynyl]phenyl\}-3-(trimethylsilyl)-2-propyn-1-ol 


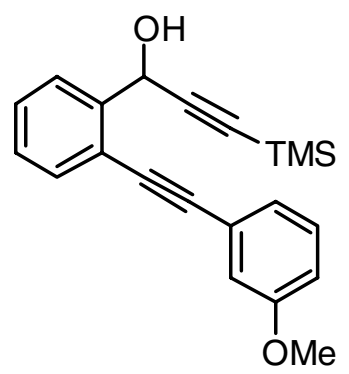

Clear oil; ${ }^{1} \mathrm{H}$ NMR $\left(\mathrm{CDCl}_{3}\right) \delta: 7.72(\mathrm{~d}, J=7.4 \mathrm{~Hz}, 1 \mathrm{H}, \mathrm{Ar} H), 7.55(\mathrm{~d}, J=7.4 \mathrm{~Hz}, 1 \mathrm{H}, \operatorname{Ar} H)$, 7.44-7.20 (m, 3H, ArH), 7.15 (d, J= 7.5 Hz, 1H, ArH), 7.06 (s, 1H, ArH), 6.91 (d, J= 7.4 $\mathrm{Hz}, 1 \mathrm{H}, \mathrm{ArH}), 5.93(\mathrm{~d}, J=3.9 \mathrm{~Hz}, 1 \mathrm{H}, \mathrm{ArCH}), 3.82$ (s, 3H, OCH $H_{3}, 2.69$ (d, J= $3.9 \mathrm{~Hz}, 1 \mathrm{H}$, $\mathrm{OH}), 0.17$ (s, 9H, $\left.\mathrm{Si}\left(\mathrm{CH}_{3}\right)_{3}\right) ;{ }^{13} \mathrm{C} \mathrm{NMR} / \mathrm{DEPT}\left(\mathrm{CDCl}_{3}\right) \delta: 159.0(\mathrm{C}), 141.59(\mathrm{C}), 132.1$ $(\mathrm{CH}), 129.2(\mathrm{CH}), 128.7(\mathrm{CH}), 128.0(\mathrm{CH}), 126.4(\mathrm{CH}), 124.0(\mathrm{CH}), 123.6(C), 121.2$ $(C H), 116.1(C), 114.9(C H), 104.6(C), 94.7(C), 90.9(C), 86.3(C), 63.2(C H), 55.0$ $\left(\mathrm{CH}_{3}\right),-0.4\left(\mathrm{Si}\left(\mathrm{CH}_{3}\right)_{3}\right)$; MS (70 eV) m/z (\%): $334\left(\mathrm{M}^{+}, 7\right), 319$ (25), 303 (11), 202 (15), 73 (100).

1-\{2-[2-(3-methoxyphenyl)-1-ethynyl]phenyl\}-3-(trimethylsilyl)propynone $\mathbf{1 g}$

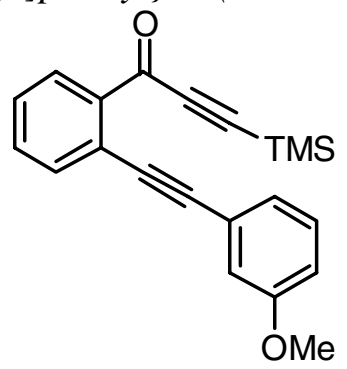

Clear oil; ${ }^{1} \mathrm{H}$ NMR $\left(\mathrm{CDCl}_{3}\right) \delta: 8.14(\mathrm{~d}, J=7.5 \mathrm{~Hz}, 1 \mathrm{H}, \mathrm{Ar} H), 7.65(\mathrm{~d}, J=7.3 \mathrm{~Hz}, 1 \mathrm{H}, \mathrm{Ar} H)$, $7.54(\mathrm{t}, J=7.3 \mathrm{~Hz}, 1 \mathrm{H}, \operatorname{ArH}), 7.45(\mathrm{t}, J=7.3 \mathrm{~Hz}, 1 \mathrm{H}, \operatorname{Ar} H), 7.31-7.18(\mathrm{~m}, 2 \mathrm{H}, \operatorname{Ar} H), 7.14$ (broad s, 1H, $\operatorname{ArH}), 6.91(\mathrm{~d}, J=7.5 \mathrm{~Hz}, 1 \mathrm{H}, \operatorname{ArH}), 3.84\left(\mathrm{~s}, 3 \mathrm{H}, \mathrm{OCH}_{3}\right), 0.26(\mathrm{~s}, 9 \mathrm{H}$, $\left.\mathrm{Si}\left(\mathrm{CH}_{3}\right)_{3}\right) ;{ }^{13} \mathrm{C}$ NMR/DEPT $\left(\mathrm{CDCl}_{3}\right) \delta: 176.9(\mathrm{CO}), 159.2(\mathrm{C}), 137.6(\mathrm{C}), 134.1(\mathrm{CH})$, $132.5(\mathrm{CH}), 132.0(\mathrm{CH}), 129.2(\mathrm{CH}), 127.8(\mathrm{CH}), 124.4(\mathrm{CH}), 124.0(C), 122.7(C), 116.3$ $(\mathrm{CH}), 115.3(\mathrm{CH}), 101.5(C), 100.3(C), 95.0(C), 87.9(C), 55.1\left(C_{3}\right),-0.9\left(\mathrm{Si}\left(\mathrm{CH}_{3}\right)_{3}\right)$; MS (70 eV) m/z (\%): $332\left(\mathrm{M}^{+}, 24\right), 317$ (66), 289 (41), 259 (38), 215 (54), 73 (100).

3-\{2-[1-Hydroxy-3-(trimethylsilyl)-2-propynyl]-phenylethynyl\}-phenol<smiles>CC#CC(O)c1ccccc1C#Cc1cccc(O)c1</smiles>

Clear oil; ${ }^{1} \mathrm{H}$ NMR $\left(\mathrm{CDCl}_{3}\right) \delta: 7.81(\mathrm{~d}, J=7.5 \mathrm{~Hz}, 1 \mathrm{H}, \mathrm{ArH}), 7.60(\mathrm{dd}, J=7.3,1.2 \mathrm{~Hz}, 1 \mathrm{H}$, $\operatorname{ArH}), 7.49-7.07(\mathrm{~m}, 5 \mathrm{H}, \operatorname{ArH}), 6.91(\mathrm{ddd}, J=7.8,2.5,1.3 \mathrm{~Hz}, 1 \mathrm{H}, \operatorname{ArH}), 6.04(\mathrm{~s}, 1 \mathrm{H}$, $\mathrm{ArCH}), 0.24\left(\mathrm{~s}, 9 \mathrm{H}, \mathrm{Si}\left(\mathrm{CH}_{3}\right)_{3}\right) ;{ }^{13} \mathrm{C} \mathrm{NMR} / \mathrm{DEPT}\left(\mathrm{CDCl}_{3}\right) \delta$ : $155.6(\mathrm{C}), 141.4(\mathrm{C}), 132.3$ $(\mathrm{CH}), 129.6(\mathrm{CH}), 128.9(\mathrm{CH}), 128.4(\mathrm{CH}), 126.9(\mathrm{CH}), 123.8(\mathrm{CH}), 123.6(C), 121.6(C)$, $118.3(\mathrm{CH}), 116.3(\mathrm{CH}), 103.9(C), 94.8(C), 92.1(C), 86.2(C), 63.7(C \mathrm{H}),-0.3\left(\mathrm{Si}\left(\mathrm{CH}_{3}\right)_{3}\right)$; 
MS (70 eV) m/z (\%): $320\left(\mathrm{M}^{+}, 28\right), 305$ (91), 303 (41), 231 (100), 202 (68); HRMS $\left(\mathrm{C}_{20} \mathrm{H}_{20} \mathrm{O}_{2} \mathrm{Si}\right)$ : calc. 320.1232; found, 320.1232 .

1-\{2-[2-(3-hydroxyphenyl)-1-ethynyl]phenyl\}-3-(trimethylsilyl)propynone $\mathbf{1 h}$<smiles>CC#CC(=O)c1ccccc1C#Cc1cccc(O)c1</smiles>

Clear oil; ${ }^{1} \mathrm{H}$ NMR $\left(\mathrm{CDCl}_{3}\right) \delta: 8.15(\mathrm{~d}, \mathrm{~J}=7.3 \mathrm{~Hz}, 1 \mathrm{H}, \mathrm{ArH}), 7.64-7.41(\mathrm{~m}, 3 \mathrm{H}, \mathrm{ArH}), 7.22-$ $7.11(\mathrm{~m}, 3 \mathrm{H}, \operatorname{ArH}), 6.88(\mathrm{~d}, \mathrm{~J}=7.3,1 \mathrm{H}, \mathrm{ArH}), 6.26$ (broad s, 1H, OH), $0.25(\mathrm{~s}, 9 \mathrm{H}$, $\left.\mathrm{Si}\left(\mathrm{CH}_{3}\right)_{3}\right) ;{ }^{13} \mathrm{C}$ NMR/DEPT $\left(\mathrm{CDCl}_{3}\right)$ \&: $177.6(\mathrm{CO}), 155.6(\mathrm{C}), 137.5(\mathrm{C}), 134.2(\mathrm{CH})$, $132.7 \mathrm{CH}), 132.2(\mathrm{CH}), 129.5(\mathrm{CH}), 127.9(\mathrm{CH}), 124.3(\mathrm{CH}), 124.1(\mathrm{C}), 122.9(\mathrm{C}), 118.6$ $(\mathrm{CH}), 116.4(\mathrm{CH}), 101.5(\mathrm{C}), 101.4(\mathrm{C}), 95.2(\mathrm{C}), 87.9(\mathrm{C}),-0.9\left(\mathrm{Si}\left(\mathrm{CH}_{3}\right)_{3}\right)$; $\mathrm{MS}(70 \mathrm{eV})$ $\mathrm{m} / z$ (\%): $318\left(\mathrm{M}^{+}, 21\right), 303$ (64), 287 (49), 73 (100). HRMS $\left(\mathrm{C}_{20} \mathrm{H}_{18} \mathrm{O}_{2} \mathrm{Si}\right)$ : calc. 318.1076; found, 318.1073.

1-\{2-[2-(2-methylphenyl)-1-ethynyl]phenyl\}-3-(trimethylsilyl)-2-propyn-1-ol<smiles>CC#CC(O)c1ccccc1C#Cc1ccccc1C</smiles>

Clear oil; ${ }^{1} \mathrm{H}$ NMR $\left(\mathrm{CDCl}_{3}\right) \delta: 7.74(\mathrm{~d}, J=9.0 \mathrm{~Hz}, 1 \mathrm{H}, \mathrm{ArH}), 7.60-7.48(\mathrm{~m}, 2 \mathrm{H}, \mathrm{ArH})$, 7.44$7.12(\mathrm{~m}, 5 \mathrm{H}, \mathrm{ArH}), 5.96$ (broad s, $1 \mathrm{H}, \mathrm{ArCH}), 2.55$ (s, 3H, $\left.\mathrm{CH}_{3}\right), 0.18$ (s, 9H, $\left.\mathrm{Si}\left(\mathrm{CH}_{3}\right)_{3}\right)$; ${ }^{13} \mathrm{C}$ NMR/DEPT $\left(\mathrm{CDCl}_{3}\right) \delta$ : $141.6(\mathrm{C}), 140.0(\mathrm{C}), 132.3(\mathrm{CH}), 132.0(\mathrm{CH}), 129.5(\mathrm{CH})$, $128.7(\mathrm{CH}), 128.6(\mathrm{CH}), 128.2(\mathrm{CH}), 126.6(\mathrm{CH}), 125.6(\mathrm{CH}), 122.5(C), 121.7(\mathrm{C}), 104.3$ (C), $93.9(C), 91.4(C), 90.3(C), 63.5(C \mathrm{H}), 20.8\left(\mathrm{CH}_{3}\right),-0.3\left(\mathrm{Si}\left(\mathrm{CH}_{3}\right)_{3}\right)$; MS $(70 \mathrm{eV}) \mathrm{m} / \mathrm{z}$ (\%): $318\left(\mathrm{M}^{+}, 3\right), 303(13), 226(26), 211(23), 73$ (100).

1-\{2-[2-(2-methylphenyl)-1-ethynyl]phenyl\}-3-(trimethylsilyl)propynone $\mathbf{1 i}$<smiles>CC#CC(=O)c1ccccc1C#Cc1ccccc1C</smiles>

Clear oil; ${ }^{1} \mathrm{H}$ NMR $\left(\mathrm{CDCl}_{3}\right) \delta: 8.17(\mathrm{~d}, J=7.4 \mathrm{~Hz}, 1 \mathrm{H}, \mathrm{Ar} H), 7.65(\mathrm{~d}, J=7.2 \mathrm{~Hz}, 1 \mathrm{H}, \operatorname{Ar} H)$, 7.61-7.39 (m, 4H, $\mathrm{ArH}), 7.28-7.12$ (m, 2H, $\mathrm{ArH}), 2.60$ (s, 3H, $\left.\mathrm{CH}_{3}\right), 0.26$ (s, 9H, $\left.\mathrm{Si}\left(\mathrm{CH}_{3}\right)_{3}\right)$; ${ }^{13} \mathrm{C}$ NMR/DEPT $\left(\mathrm{CDCl}_{3}\right)$ 8: $176.8(\mathrm{CO}), 140.8(\mathrm{C}), 137.2(\mathrm{C}), 134.4(\mathrm{CH}), 132.5(\mathrm{CH})$, $132.4(\mathrm{CH}), 132.2(\mathrm{CH}), 129.4(\mathrm{CH}), 128.7(\mathrm{CH}), 127.7(\mathrm{CH}), 125.4(\mathrm{CH}), 123.1(\mathrm{C})$, $122.9(C), 101.5(C), 100.3(C), 94.4(C), 91.8(C), 20.7\left(\mathrm{CH}_{3}\right),-0.8\left(\mathrm{Si}\left(\mathrm{CH}_{3}\right)_{3}\right)$; MS $(70$ eV) $\mathrm{m} / \mathrm{z}(\%): 316\left(\mathrm{M}^{+}, 13\right), 302$ (27), 301 (100), 285 (42), 273 (45), 215 (33), 189 (34). 
1-\{2-[2-(2-methoxyphenyl)-1-ethynyl]phenyl\}-3-(trimethylsilyl)-2-propyn-1-ol<smiles>COc1ccccc1C#Cc1ccccc1C(O)C#CC(C)(C)C</smiles>

Clear oil; ${ }^{1} \mathrm{H}$ NMR $\left(\mathrm{CDCl}_{3}\right) \delta: 7.68(\mathrm{~d}, J=7.5 \mathrm{~Hz}, 1 \mathrm{H}, \operatorname{Ar} H), 7.54(\mathrm{~d}, J=7.9 \mathrm{~Hz}, 1 \mathrm{H}, \operatorname{Ar} H)$, $7.49(\mathrm{~d}, J=7.5 \mathrm{~Hz}, 1 \mathrm{H}, \operatorname{Ar} H), 7.40-7.27(\mathrm{~m}, 3 \mathrm{H}, \mathrm{Ar} H), 7.01-6.87$ (m, 2H, $\operatorname{Ar} H), 5.86(\mathrm{~d}, J=$ $7.5 \mathrm{~Hz}, 1 \mathrm{H}, \operatorname{ArCH}), 4.25(\mathrm{~d}, J=7.5 \mathrm{~Hz}, 1 \mathrm{H}, \mathrm{OH}), 3.94\left(\mathrm{~s}, 3 \mathrm{H}, \mathrm{OCH}_{3}\right), 0.15(\mathrm{~s}, 9 \mathrm{H}$, $\left.\mathrm{Si}\left(\mathrm{CH}_{3}\right)_{3}\right) ;{ }^{13} \mathrm{C}$ NMR/DEPT $\left(\mathrm{CDCl}_{3}\right)$ \&: $159.9(\mathrm{C}), 142.5(\mathrm{C}), 132.3(\mathrm{CH}), 131.8(\mathrm{CH})$, $130.0(\mathrm{CH}), 128.4(\mathrm{CH}), 128.0(\mathrm{CH}), 127.1(\mathrm{CH}), 121.7(\mathrm{C}), 120.6(\mathrm{CH}), 111.8(\mathrm{C}), 110.3$ $(\mathrm{CH}), 104.0(\mathrm{C}), 91.6(\mathrm{C}), 91.4(\mathrm{C}), 91.3(\mathrm{C}), 64.0(\mathrm{CH}), 55.7\left(\mathrm{CH}_{3}\right),-0.3\left(\mathrm{Si}\left(\mathrm{CH}_{3}\right)_{3}\right)$; $\mathrm{MS}$ (70 eV) $\mathrm{m} / z$ (\%): $334\left(\mathrm{M}^{+}, 3\right), 319(14), 303$ (12), 215 (13), 108 (13), 73 (100). 1-\{2-[2-(2-methoxyphenyl)-1-ethynyl]phenyl\}-3-(trimethylsilyl)propynone $\mathbf{1} \mathbf{j}$<smiles>CC#CC(=O)c1ccccc1C#Cc1ccccc1OC</smiles>

Clear oil; ${ }^{1} \mathrm{H}$ NMR $\left(\mathrm{CDCl}_{3}\right) \delta: 8.13(\mathrm{~d}, J=7.5 \mathrm{~Hz}, 1 \mathrm{H}, \operatorname{Ar} H), 7.68(\mathrm{~d}, J=7.1 \mathrm{~Hz}, 1 \mathrm{H}, \operatorname{Ar} H)$, $7.61(\mathrm{~d}, J=7.1 \mathrm{~Hz}, 1 \mathrm{H}, \operatorname{Ar} H), 7.52(\mathrm{t}, J=7.2 \mathrm{~Hz}, 1 \mathrm{H}, \operatorname{Ar} H), 7.42(\mathrm{~d}, J=7.2 \mathrm{~Hz}, 1 \mathrm{H}, \operatorname{Ar} H)$, $7.31(\mathrm{~d}, J=7.8 \mathrm{~Hz}, 1 \mathrm{H}, \mathrm{ArH}), 6.99-6.85(\mathrm{~m}, 2 \mathrm{H}, \mathrm{ArH}), 3.93$ (s, 3H, OCH $)_{3}, 0.24$ (s, 9H, $\left.\mathrm{Si}\left(\mathrm{CH}_{3}\right)_{3}\right) ;{ }^{13} \mathrm{C}$ NMR/DEPT $\left(\mathrm{CDCl}_{3}\right)$ \&: $176.9(\mathrm{CO}), 160.1(\mathrm{C}), 137.4(\mathrm{C}), 134.3(\mathrm{CH})$, $134.0(\mathrm{CH}), 132.4(\mathrm{CH}), 131.9(\mathrm{CH}), 130.1(\mathrm{CH}), 127.6(\mathrm{CH}), 123.1(C), 120.4(\mathrm{CH})$, $112.4(C), 110.6(\mathrm{CH}), 101.6(C), 100.1(C), 92.0(C), 91.9(C), 55.8\left(C_{3}\right),-0.9$ $\left(\mathrm{Si}\left(\mathrm{CH}_{3}\right)_{3}\right) ; \mathrm{MS}(70 \mathrm{eV}) \mathrm{m} / z(\%): 332\left(\mathrm{M}^{+}, 13\right), 317$ (32), 287 (23), 215 (35), 73 (100), 58 (60).

1-\{2-[2-(2-t-butylphenyl)-1-ethynyl]phenyl\}-3-(trimethylsilyl)-2-propyn-1-ol<smiles>CC#CC(O)c1ccccc1C#Cc1ccccc1C(C)(C)C</smiles>

Clear oil; ${ }^{1} \mathrm{H}$ NMR $\left(\mathrm{CDCl}_{3}\right) \delta: 7.73(\mathrm{dd}, \mathrm{J}=7.9,1.7,1 \mathrm{H}, \mathrm{Ar} H), 7.63(\mathrm{dd}, J=7.0,1.7 \mathrm{~Hz}, 1 \mathrm{H}$, $\operatorname{ArH}), 7.56(\mathrm{dd}, \mathrm{J}=7.0,1.7,1 \mathrm{H}, \operatorname{ArH}), 7.43-7.26(\mathrm{~m}, 4 \mathrm{H}, \operatorname{ArH}), 7.19(\mathrm{t}, J=7.0 \mathrm{~Hz}, 1 \mathrm{H}$, $\mathrm{ArH}), 5.97(\mathrm{~d}, \mathrm{~J}=5.2 \mathrm{~Hz}, 1 \mathrm{H}, \mathrm{ArCH}), 5.60(\mathrm{~d}, \mathrm{~J}=5.2 \mathrm{~Hz}, 1 \mathrm{H}, \mathrm{OH}), 1.60\left(\mathrm{~s}, 9 \mathrm{H}, \mathrm{C}\left(\mathrm{CH}_{3}\right)_{3}\right)$, 0.20 (s, 9H, $\left.\mathrm{Si}\left(\mathrm{CH}_{3}\right)_{3}\right) ;{ }^{13} \mathrm{C}$ NMR/DEPT $\left(\mathrm{CDCl}_{3}\right) \delta: 151.3(\mathrm{C}), 141.5(\mathrm{C}), 135.4(\mathrm{CH}), 131.9$ $(\mathrm{CH}), 128.7(\mathrm{CH}), 128.6(\mathrm{CH}), 128.3(\mathrm{CH}), 126.7(\mathrm{CH}), 125.6(\mathrm{CH}), 125.5(\mathrm{CH}), 122.1$ 
(C), $\left.121.0(C), 104.4(C), 96.5(C), 92.0(C), 91.5(C), 63.6(C H), 35.8(C), 30.1\left(\mathrm{C}_{(C H}\right)_{3}\right)$, $-0.25\left(\mathrm{Si}\left(\mathrm{CH}_{3}\right)_{3}\right) ; \mathrm{MS}(70 \mathrm{eV}) \mathrm{m} / z(\%): 360\left(\mathrm{M}^{+}, 11\right), 304(100), 289$ (94); HRMS $\left(\mathrm{C}_{24} \mathrm{H}_{28} \mathrm{OSi}\right)$ : calc. 360.1909 ; found, 360.1908 .

1-\{2-[2-(2-t-butylphenyl)-1-ethynyl]phenyl\}-3-(trimethylsilyl)propynone $\mathbf{1 k}$

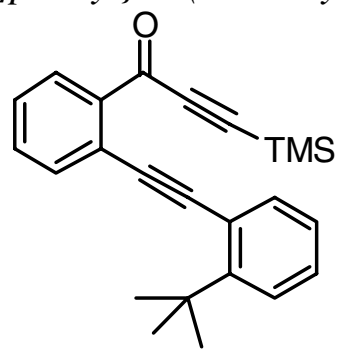

Clear oil; ${ }^{1} \mathrm{H}$ NMR $\left(\mathrm{CDCl}_{3}\right) \delta: 7.75(\mathrm{~d}, J=7.3,1 \mathrm{H}, \mathrm{Ar} H), 7.65(\mathrm{~d}, J=7.3,1 \mathrm{H}, \mathrm{Ar} H), 7.55$ $(\mathrm{t}, J=7.3,1 \mathrm{H}, \operatorname{Ar} H), 7.47-7.16,(\mathrm{~m}, 5 \mathrm{H}, \operatorname{Ar} H), 1.58\left(\mathrm{~s}, 9 \mathrm{H}, \mathrm{C}\left(\mathrm{CH}_{3}\right)_{3}\right), 0.26(\mathrm{~s}, 9 \mathrm{H}$, $\left.\mathrm{Si}\left(\mathrm{CH}_{3}\right)_{3}\right) ;{ }^{13} \mathrm{C}$ NMR/DEPT $\left(\mathrm{CDCl}_{3}\right) \delta: 177.0(\mathrm{CO}), 151.7(\mathrm{C}), 137.2(\mathrm{C}), 136.0(\mathrm{CH})$, $133.5(\mathrm{CH}), 132.6(\mathrm{CH}), 132.2(\mathrm{CH}), 128.7(\mathrm{CH}), 127.7(\mathrm{CH}), 125.6(\mathrm{CH}), 125.4(\mathrm{CH})$, 123.6 (C), $121.5(C), 101.6(C), 100.4(C), 96.9(C), 93.7(C), 35.9(C), 30.3\left(\mathrm{C}_{\left.\left(C \mathrm{H}_{3}\right)_{3}\right)}\right)$, $0.75\left(\mathrm{Si}\left(\mathrm{CH}_{3}\right)_{3}\right)$; MS (70 eV) $\mathrm{m} / z(\%): 358\left(\mathrm{M}^{+}, 33\right), 343$ (89), 253 (82), 135 (100); HRMS $\left(\mathrm{C}_{24} \mathrm{H}_{26} \mathrm{OSi}\right)$ : calc. 358.1758 ; found, 358.1760 .

1-\{2-[2-(2-trimethylsilylphenyl)-1-ethynyl]phenyl\}-3-(trimethylsilyl)-2-propyn-1-ol<smiles>CC#CC(O)c1ccccc1C#Cc1ccccc1S(C)(=O)=O</smiles>

Clear oil; ${ }^{1} \mathrm{H}$ NMR $\left(\mathrm{CDCl}_{3}\right) \delta$ : $7.75(\mathrm{~d}, J=7.5 \mathrm{~Hz}, 1 \mathrm{H}, \mathrm{Ar} H), 7.63-7.60(\mathrm{~m}, 1 \mathrm{H}, \mathrm{ArH}), 7.56-$ $7.53(\mathrm{~m}, 2 \mathrm{H}, \mathrm{Ar} H), 7.43-7.32(\mathrm{~m}, 4 \mathrm{H}, \mathrm{Ar} H), 5.96(\mathrm{~d}, J=5.0 \mathrm{~Hz}, 1 \mathrm{H}, \mathrm{ArCH}), 2.65(\mathrm{~d}, J=5.0$ $\mathrm{Hz}, 1 \mathrm{H}, \mathrm{OH}), 0.44$ (s, 9H, Si $\left.\left(\mathrm{CH}_{3}\right)_{3}\right), 0.18\left(\mathrm{~s}, 9 \mathrm{H}, \mathrm{Si}\left(\mathrm{CH}_{3}\right)_{3}\right) ;{ }^{13} \mathrm{C}$ NMR/DEPT $\left(\mathrm{CDCl}_{3}\right) \delta$ : $142.1(\mathrm{C}), 141.8(\mathrm{C}), 134.0(\mathrm{CH}), 132.8(\mathrm{CH}), 131.9(\mathrm{CH}), 128.8(\mathrm{CH}), 128.7(\mathrm{CH}), 128.4$ $(\mathrm{CH}), 127.9(\mathrm{C}), 127.8(\mathrm{CH}), 126.9(\mathrm{CH}), 121.8(\mathrm{C}), 104.3(\mathrm{C}), 96.5(\mathrm{C}), 91.5(\mathrm{C}), 89.1$ (C), $63.6(\mathrm{CH}),-0.2\left(\mathrm{Si}\left(\mathrm{CH}_{3}\right)_{3}\right),-0.9\left(\mathrm{Si}\left(\mathrm{CH}_{3}\right)_{3}\right) ; \mathrm{MS}(70 \mathrm{eV}) \mathrm{m} / \mathrm{z}(\%): 376\left(\mathrm{M}^{+}, 29\right), 303$ (47), 287 (54), 271 (52), 73 (100); HRMS $\left(\mathrm{C}_{23} \mathrm{H}_{28} \mathrm{OSi}_{2}\right)$ : calc. 376.1678; found, 376.1671. 1-\{2-[2-(2-trimethylsilylphenyl)-1-ethynyl]phenyl\}-3-(trimethylsilyl)-2-propyn-1-one $\mathbf{1 1}$

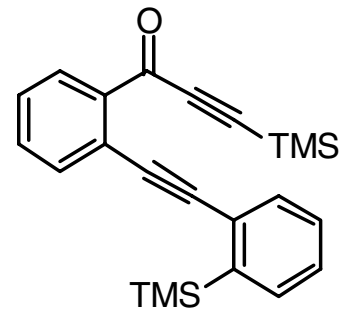

Clear oil; ${ }^{1} \mathrm{H}$ NMR $\left(\mathrm{CDCl}_{3}\right) \delta: 8.15(\mathrm{~d}, J=7.9 \mathrm{~Hz}, 1 \mathrm{H}, \mathrm{Ar} H), 7.73(\mathrm{dd}, J=6.6,2.0 \mathrm{~Hz}, 1 \mathrm{H}$, $\mathrm{ArH})$, 7.63-7.23 (m, 6H, $\mathrm{ArH}), 0.45\left(\mathrm{~s}, 9 \mathrm{H}, \mathrm{Si}\left(\mathrm{CH}_{3}\right)_{3}\right), 0.27$ (s, 9H, $\left.\mathrm{Si}\left(\mathrm{CH}_{3}\right)_{3}\right) ;{ }^{13} \mathrm{C}$ NMR/DEPT $\left(\mathrm{CDCl}_{3}\right)$ 8: $177.0(\mathrm{CO}), 142.3(\mathrm{C}), 137.6(\mathrm{C}), 133.8(\mathrm{CH}), 133.5(\mathrm{CH}), 133.4$ 
$(\mathrm{CH}), 132.6(\mathrm{CH}), 132.1(\mathrm{CH}), 128.8(\mathrm{CH}), 128.3(\mathrm{C}), 127.8(\mathrm{CH}), 127.7(\mathrm{CH}), 123.1(\mathrm{C})$, 101.6 (C), $\left.100.5(C), 97.0(C), 90.7(C),-0.8\left(\mathrm{Si}\left(\mathrm{CH}_{3}\right)_{3}\right),-0.9\left(\mathrm{Si}_{(\mathrm{CH}}\right)_{3}\right)$; MS $(70 \mathrm{eV}) \mathrm{m} / \mathrm{z}$ (\%): $374\left(\mathrm{M}^{+}, 5\right), 359$ (14), 285 (11), 73 (100); HRMS $\left(\mathrm{C}_{23} \mathrm{H}_{26} \mathrm{OSi}_{2}\right)$ : calc. 374.1522; found, 376.1529 .

1-\{2-[2-(4-methoxy-2-methylphenyl)-1-ethynyl]phenyl\}-3-(trimethylsilyl)-2-propyn-1-ol<smiles>COc1ccc(C#Cc2ccccc2C(O)C#CC(C)(C)C)c(C)c1</smiles>

Yellowish oil; ${ }^{1} \mathrm{H}$ NMR $\left(\mathrm{CDCl}_{3}\right) \delta: 7.73(\mathrm{dd}, J=7.2,1.9 \mathrm{~Hz}, 1 \mathrm{H}, \mathrm{Ar} H), 7.54(\mathrm{dd}, J=7.2$, $1.9 \mathrm{~Hz}, 1 \mathrm{H}, \operatorname{Ar} H), 7.45(\mathrm{~d}, J=8.5 \mathrm{~Hz}, 1 \mathrm{H}, \operatorname{Ar} H), 7.40-7.26(\mathrm{~m}, 2 \mathrm{H}, \operatorname{Ar} H), 6.78(\mathrm{~d}, J=2.8$ $\mathrm{Hz}, 1 \mathrm{H}, \mathrm{Ar} H), 6.72(\mathrm{dd}, J=8.5,2.8 \mathrm{~Hz}, 1 \mathrm{H}, \mathrm{Ar} H), 5.95(\mathrm{~d}, J=5.7 \mathrm{~Hz}, 1 \mathrm{H}, \operatorname{ArCH}), 3.81(\mathrm{~s}$, $\left.3 \mathrm{H}, \mathrm{OCH}_{3}\right), 2.53\left(\mathrm{~s}, 3 \mathrm{H}, \mathrm{CH}_{3}\right), 0.18\left(\mathrm{~s}, 9 \mathrm{H}, \mathrm{Si}\left(\mathrm{CH}_{3}\right)_{3}\right) ;{ }^{13} \mathrm{C} \mathrm{NMR} / \mathrm{DEPT}\left(\mathrm{CDCl}_{3}\right) \delta: 159.8$ $(C), 141.9(C), 141.4(C), 133.4(\mathrm{CH}), 132.2(\mathrm{CH}), 128.3(\mathrm{CH}), 128.1(\mathrm{CH}), 126.6(\mathrm{CH})$, $122.1(C), 115.1(\mathrm{CH}), 114.9(C), 111.3(\mathrm{CH}), 104.4(C), 94.0(C), 91.3(C), 89.0(C), 63.6$ $(\mathrm{CH}), 55.1\left(\mathrm{CH}_{3}\right), 21.1\left(\mathrm{CH}_{3}\right),-0.25\left(\mathrm{Si}\left(\mathrm{CH}_{3}\right)_{3}\right) ; \mathrm{MS}(70 \mathrm{eV}) \mathrm{m} / z(\%): 348\left(\mathrm{M}^{+}, 28\right), 333$ (100).

1-\{2-[2-(2,4-dimethoxyphenyl)-1-ethynyl]phenyl\}-3-(trimethylsilyl)-2-propyn-1-ol

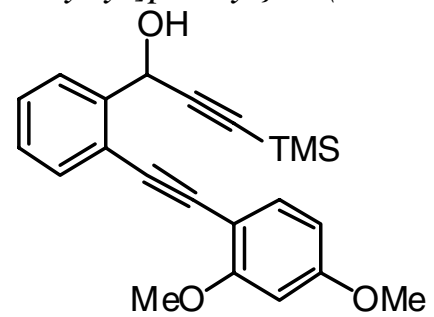

Yellowish oil; ${ }^{1} \mathrm{H}$ NMR $\left(\mathrm{CDCl}_{3}\right)$ 8: 7.70-7.66 (m, $\left.1 \mathrm{H}, \mathrm{ArH}\right), 7.55-7.50(\mathrm{~m}, 1 \mathrm{H}, \mathrm{ArH})$, 7.44$7.29(\mathrm{~m}, 4 \mathrm{H}, \operatorname{Ar} H), 6.48(\mathrm{~s}, 1 \mathrm{H}, \operatorname{Ar} H), 5.86(\mathrm{~d}, J=6.9 \mathrm{~Hz}, 1 \mathrm{H}, \operatorname{ArCH}), 4.20(\mathrm{~d}, J=6.9 \mathrm{~Hz}$, $1 \mathrm{H}, \mathrm{OH}), 3.92\left(\mathrm{~s}, 3 \mathrm{H}, \mathrm{OCH}_{3}\right), 3.84\left(\mathrm{~s}, 3 \mathrm{H}, \mathrm{OCH}_{3}\right), 0.16$ (s, 9H, $\left.\mathrm{Si}\left(\mathrm{CH}_{3}\right)_{3}\right) ;{ }^{13} \mathrm{C}$ NMR/DEPT

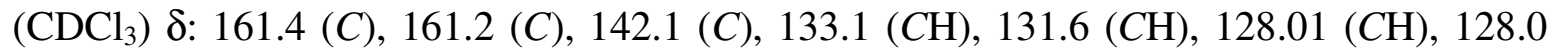
$(\mathrm{CH}), 127.0(\mathrm{CH}), 122.1(\mathrm{C}), 120.0(\mathrm{C}), 104.9(\mathrm{CH}), 104.4(\mathrm{C}), 98.2(\mathrm{CH}), 91.8(\mathrm{C}), 91.4$ (C), $90.0(C), 64.0(\mathrm{CH}), 55.7\left(\mathrm{CH}_{3}\right), 55.3\left(\mathrm{CH}_{3}\right),-0.3\left(\mathrm{Si}\left(\mathrm{CH}_{3}\right)_{3}\right) ; \mathrm{MS}(70 \mathrm{eV}) \mathrm{m} / \mathrm{z}(\%): 364$ $\left(\mathrm{M}^{+}, 12\right), 349$ (66), 333 (12), 291 (12), 138 (100); HRMS $\left(\mathrm{C}_{22} \mathrm{H}_{24} \mathrm{O}_{3} \mathrm{Si}\right)$ : calc. 364.5096; found, 364.5099 .

1-\{2-[2-(2,4-dimethoxyphenyl)-1-ethynyl]phenyl\}-3-(trimethylsilyl)propynone $\mathbf{1 n}$

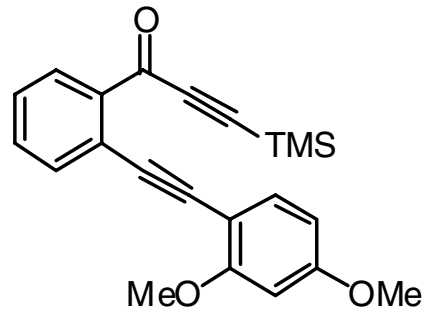


Yellowish oil; ${ }^{1} \mathrm{H}$ NMR $\left(\mathrm{CDCl}_{3}\right) \delta: 8.11(\mathrm{~d}, J=7.3 \mathrm{~Hz}, 1 \mathrm{H}, \mathrm{ArH}), 7.63(\mathrm{~d}, J=7.3 \mathrm{~Hz}, 1 \mathrm{H}$, $\operatorname{ArH}), 7.53(\mathrm{~d}, J=6.9 \mathrm{~Hz}, 1 \mathrm{H}, \mathrm{ArH}), 7.49$ (t, $J=7.3 \mathrm{~Hz}, 1 \mathrm{H}, \operatorname{ArH}), 7.38(\mathrm{t}, J=7.3 \mathrm{~Hz}, 1 \mathrm{H}$, $\left.\mathrm{ArH}), 6.49-6.43(\mathrm{~m}, 2 \mathrm{H}, \mathrm{ArH}), 3.90\left(\mathrm{~s}, 3 \mathrm{H}, \mathrm{CH}_{3}\right), 3.80\left(\mathrm{~s}, 3 \mathrm{H}, \mathrm{CH}_{3}\right), 0.24 \mathrm{~s}, 9 \mathrm{H}, \mathrm{Si}\left(\mathrm{CH}_{3}\right)_{3}\right)$; ${ }^{13} \mathrm{C}$ NMR/DEPT $\left(\mathrm{CDCl}_{3}\right)$ $\delta: 177.0(C \mathrm{O}), 161.5(C), 161.4(C), 137.1(C), 135.0(C \mathrm{H})$, 134.1 $(\mathrm{CH}), 132.4(\mathrm{CH}), 132.0(\mathrm{CH}), 127.1(\mathrm{CH}), 123.6(C), 104.9(C \mathrm{H}), 101.6(C), 99.9$ $(C), 98.2(\mathrm{CH}), 92.3(C), 91.1(C), 55.8\left(\mathrm{CH}_{3}\right), 55.3\left(\mathrm{CH}_{3}\right),-0.8\left(\mathrm{Si}\left(\mathrm{CH}_{3}\right)_{3}\right)$; $\mathrm{MS}(70 \mathrm{eV}) \mathrm{m} / z$ (\%): $362\left(\mathrm{M}^{+}, 35\right), 347$ (100), 289 (31). HRMS $\left(\mathrm{C}_{22} \mathrm{H}_{22} \mathrm{O}_{3} \mathrm{Si}\right)$ : calc. 362.1338; found, 362.1341 .

1-\{2-[2-(2,4-dimethylphenyl)-1-ethynyl]phenyl\}-3-(trimethylsilyl)-2-propyn-1-ol

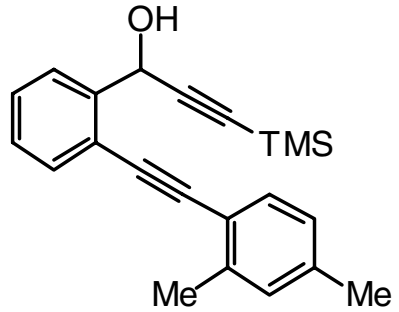

Clear oil; ${ }^{1} \mathrm{H}$ NMR $\left(\mathrm{CDCl}_{3}\right) \delta: 7.73(\mathrm{dd}, J=7.6,1.5 \mathrm{~Hz}, 1 \mathrm{H}, \mathrm{ArH}), 7.55(\mathrm{dd}, J=7.3,1.6 \mathrm{~Hz}$, 1H, ArH), 7.44-7.28 (m, 3H, ArH), 7.06 (broad s, 1H, ArH), 6.99 (d, J=7.8 Hz, 1H, ArH), $5.96(\mathrm{~d}, J=5.7 \mathrm{~Hz}, 1 \mathrm{H}, \mathrm{ArCH}), 2.65(\mathrm{~d}, J=5.7 \mathrm{~Hz}, 1 \mathrm{H}, \mathrm{OH}), 2.51\left(\mathrm{~s}, 3 \mathrm{H}, \mathrm{CH}_{3}\right), 2.51(\mathrm{~s}, 3 \mathrm{H}$, $\left.\mathrm{CH}_{3}\right), 0.18$ (s, 9H, $\left.\mathrm{Si}\left(\mathrm{CH}_{3}\right)_{3}\right) ;{ }^{13} \mathrm{C}$ NMR/DEPT $\left(\mathrm{CDCl}_{3}\right) \delta: 141.5(\mathrm{C}), 139.9(\mathrm{C}), 138.7(\mathrm{C})$, $132.3(\mathrm{CH}), 131.9(\mathrm{CH}), 130.3(\mathrm{CH}), 128.5(\mathrm{CH}), 128.1(\mathrm{CH}), 126.6(\mathrm{CH}), 126.4(C \mathrm{H})$, $121.9(C), 119.5(C), 104.4(C), 94.1(C), 91.3(C), 89.6(C), 63.5(C H), 21.4\left(C_{3}\right), 20.7$ $\left(\mathrm{CH}_{3}\right),-0.3\left(\mathrm{Si}\left(\mathrm{CH}_{3}\right)_{3}\right)$; MS (70 eV) m/z (\%): $332\left(\mathrm{M}^{+}, 2\right), 317$ (11), $226(32), 211$ (34), 75 (33), 73 (100).

1-\{2-[2-(2,4-dimethylphenyl)-1-ethynyl]phenyl\}-3-(trimethylsilyl)-2-propyn-1-one 10

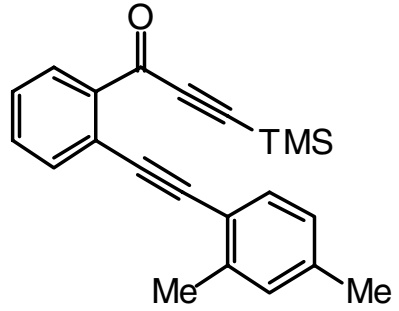

Clear oil; ${ }^{1} \mathrm{H}$ NMR $\left(\mathrm{CDCl}_{3}\right)$ $\delta: 8.16(\mathrm{dd}, J=7.8,1.6 \mathrm{~Hz}, 1 \mathrm{H}, \mathrm{ArH}), 7.65(\mathrm{dd}, J=7.6,1.6 \mathrm{~Hz}$, 1H, $\operatorname{ArH}), 7.57-7.39(\mathrm{~m}, 3 \mathrm{H}, \operatorname{Ar} H), 7.06$ (broad s, $1 \mathrm{H}, \operatorname{Ar} H), 6.98(\mathrm{~d}, J=7.8 \mathrm{~Hz}, 1 \mathrm{H}, \operatorname{Ar} H)$, 2.57 (s, 3H, $\left.\mathrm{CH}_{3}\right), 2.33$ (s, 3H, $\left.\mathrm{CH}_{3}\right), 0.26\left(\mathrm{~s}, 9 \mathrm{H}, \mathrm{Si}\left(\mathrm{CH}_{3}\right)_{3}\right) ;{ }^{13} \mathrm{C} \mathrm{NMR} / \mathrm{DEPT}\left(\mathrm{CDCl}_{3}\right) \delta$ : $176.9(C \mathrm{O}), 140.7(C), 138.9(C), 137.2(C), 134.4(C H), 132.5(C H), 132.4(C H), 132.2$ $(C H), 130.3(C H), 127.5(C H), 126.3(C H), 123.4(C), 119.9(C), 101.6(C), 100.2(C), 94.8$ (C), $91.3(C), 21.5\left(\mathrm{CH}_{3}\right), 20.7\left(\mathrm{CH}_{3}\right),-0.8\left(\mathrm{Si}\left(\mathrm{CH}_{3}\right)_{3}\right)$; $\mathrm{MS}(70 \mathrm{eV}) \mathrm{m} / z(\%): 330\left(\mathrm{M}^{+}, 16\right)$, 315 (100), 287 (38), 285 (39), 239 (39), 202 (36), 73 (88).

Preparation of 1-[(1-arylethynyl)naphthalen-2-yl]-3-(trimethylsilyl)-prop-2-yn-1-ols: 1-(Phenylethynyl)naphthalene-2-carbaldehyde. To a stirred solution of 1-bromonaphthalene-2-carbaldehyde (394 mg, $1.68 \mathrm{mmol}), \mathrm{Pd}(\mathrm{OAc})_{2}(8 \mathrm{mg}, 0.03 \mathrm{mmol}), \mathrm{CuI}(6$ $\mathrm{mg}, 0.03 \mathrm{mmol})$ and $\mathrm{PPh}_{3}(22 \mathrm{mg}, 0.08 \mathrm{mmol})$ in dry degassed $\mathrm{Et}_{3} \mathrm{~N}(10 \mathrm{~mL})$ was added phenylacetylene $(209 \mathrm{mg}, 2.01 \mathrm{mmol})$ and the whole mixture was heated at $90{ }^{\circ} \mathrm{C}$ for $6 \mathrm{~h}$. The mixture was passed through a pad of Celite to remove the solids. The filtrate was concentrated and the reidue dissolved in EtOAc, washed with 5\% aqueous $\mathrm{HCl}$ and brine, 
dried over anhydrous $\mathrm{Na}_{2} \mathrm{SO}_{4}$ and concentrated to dryness. Purification of the residue by flash chromatography on silica gel using a mixture of 95: 5 hexanes/EtOAc as eluent afforded the desired coupled product (329 mg, 76\%).

1-[(1-Phenylethynyl)naphthalen-2-yl]-3-(trimethylsilyl)-prop-2-yn-1-ol. $n$-BuLi (812 $\mu \mathrm{L}$, $1.3 \mathrm{mmol}, 1.6 \mathrm{M}$ in hexanes) was slowly added to a solution of trimethylsilylacetylene (140 $\mathrm{mg}, 1.42 \mathrm{mmol})$ in dry THF $(7 \mathrm{~mL})$ cooled at $-78{ }^{\circ} \mathrm{C}$ and the mixture was stirred for 5 minutes. This mixture was cannulated to a solution of the above aldehyde $(317 \mathrm{~g}, 1.24$ $\mathrm{mmol})$ in THF $(7 \mathrm{~mL})$ maintained at $-78{ }^{\circ} \mathrm{C}$. The resulting solution was allowed to reach room temperature and stirred for $30 \mathrm{~min}$. After removing the volatiles, the residue was solved in EtOAc $(30 \mathrm{~mL})$ and washed with brine $(3 \times 30 \mathrm{~mL})$. The organic layer was dried over anhydrous $\mathrm{Na}_{2} \mathrm{SO}_{4}$ and evaporated to dryness. The crude residue was purified by column cromatography on silica gel using 95:5 hexane/EtOAc as eluent to yield the desired alcohol (328 mg, 75\%).

\section{1-[1-(Phenylethynyl)naphthalen-2-yl]-3-(trimethylsilyl)-2-propyn-1-ol}<smiles>CC#CC(O)c1ccc2ccccc2c1C#Cc1ccccc1</smiles>

Yellow powder; mp 70-72 ${ }^{\circ} \mathrm{C} ;{ }^{1} \mathrm{H}$ NMR $\left(\mathrm{CDCl}_{3}\right) \delta: 8.50(\mathrm{~d}, J=7.5 \mathrm{~Hz}, 1 \mathrm{H}, \mathrm{ArH}), 7.93(\mathrm{~d}, J$ $=8.5 \mathrm{~Hz}, 1 \mathrm{H}, \operatorname{ArH}), 7.88-7.83(\mathrm{~m}, 2 \mathrm{H}, \operatorname{ArH}), 7.78-7.70(\mathrm{~m}, 2 \mathrm{H}, \operatorname{ArH}), 7.68-7.53(\mathrm{~m}, 2 \mathrm{H}$, $\operatorname{ArH}), 7.46-7.40(\mathrm{~m}, 3 \mathrm{H}, \operatorname{ArH}), 6.33(\mathrm{~d}, J=4.7 \mathrm{~Hz}, 1 \mathrm{H}, \operatorname{ArCH}), 3.18(\mathrm{~d}, J=4.7 \mathrm{~Hz}, 1 \mathrm{H}$, $\mathrm{OH}), 0.28\left(\mathrm{~s}, 9 \mathrm{H}, \mathrm{Si}\left(\mathrm{CH}_{3}\right)_{3}\right) ;{ }^{13} \mathrm{C} \mathrm{NMR} / \mathrm{DEPT}\left(\mathrm{CDCl}_{3}\right) \delta: 140.5(\mathrm{C}), 132.9(\mathrm{C}), 132.7(\mathrm{C})$, $131.5(2 \times \mathrm{CH}), 129.0(\mathrm{CH}), 128.6(\mathrm{CH}), 128.3(2 \times \mathrm{CH}), 128.0(\mathrm{CH}), 126.9(\mathrm{CH}), 126.5$ $(C H), 126.3(C H), 123.8(C H), 122.8(C), 118.6(C), 104.7(C), 100.2(C), 91.3(C), 84.5$ (C), $63.7(\mathrm{CH}),-0.3\left(\mathrm{Si}\left(\mathrm{CH}_{3}\right)_{3}\right)$; MS (70 eV) m/z (\%): $354\left(\mathrm{M}^{+}, 8\right), 339$ (10), 265 (16), 227 (7), 73 (100); HRMS $\left(\mathrm{C}_{24} \mathrm{H}_{22} \mathrm{OSi}\right)$ : calc. 354.1439; found, 354.1432.

\section{1-[1-(2-t-Butylphenylethynyl)naphthalen-2-yl]-3-(trimethylsilyl)-2-propyn-1-ol}

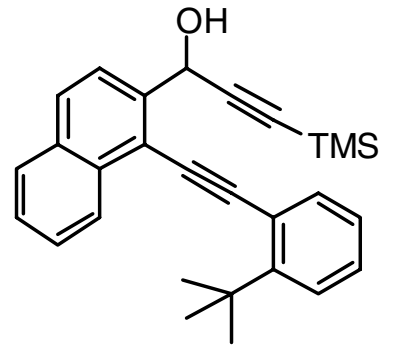

Clear oil; ${ }^{1} \mathrm{H}$ NMR $\left(\mathrm{CDCl}_{3}\right) \delta: 8.41(\mathrm{~d}, J=7.5,1 \mathrm{H}, \mathrm{Ar} H), 7.80(\mathrm{~m}, 3 \mathrm{H}, \mathrm{Ar} H), 7.68(\mathrm{dd}, J=$ 7.4, 1.6, 1H, $\operatorname{ArH}), 7.55-7.36(\mathrm{~m}, 3 \mathrm{H}, \operatorname{ArH}), 7.26(\mathrm{td}, J=7.4,1.6,1 \mathrm{H}, \operatorname{ArH}), 7.16(\mathrm{td}, J=$ 7.4, 1.6, 1H, $\operatorname{ArH}), 6.18(\mathrm{~d}, J=5.0,1 \mathrm{H}, \operatorname{ArCH}), 2.50(\mathrm{~d}, J=5.0,1 \mathrm{H}, \mathrm{OH}), 1.56(\mathrm{~s}$, $\left.\mathrm{C}\left(\mathrm{CH}_{3}\right)_{3}\right), 0.09\left(\mathrm{~s}, \mathrm{Si}\left(\mathrm{CH}_{3}\right)_{3}\right) ;{ }^{13} \mathrm{C}$ NMR/DEPT $\left(\mathrm{CDCl}_{3}\right) \delta: 151.5(C), 140.7(C), 136.0(\mathrm{CH})$, $133.6(C), 133.2(C), 129.2(C H), 128.4(C H), 127.3(C H), 126.9(C H), 126.8(C H), 125.9$ $(C \mathrm{H}), 125.8(\mathrm{CH}), 124.2(\mathrm{CH}), 121.4(C), 116.6(C), 104.8(C), 101.7(C), 91.9(C), 90.8$ 
(C), $64.0(\mathrm{C}), 36.1\left(\mathrm{C}\left(\mathrm{CH}_{3}\right)_{3}\right), 30.5\left(\mathrm{C}\left(\mathrm{CH}_{3}\right)_{3}\right),-0.01\left(\mathrm{Si}\left(\mathrm{CH}_{3}\right)_{3}\right)$; $\mathrm{MS}(70 \mathrm{eV}) \mathrm{m} / \mathrm{z}(\%): 410$ $\left(\mathrm{M}^{+}, 2\right), 276$ (13), 73 (100); HRMS $\left(\mathrm{C}_{28} \mathrm{H}_{30} \mathrm{OSi}\right):$ calc.: 410.2065, found: 410.2060.

Oxidation of 1-[(1-arylethynyl)naphthalen-2-yl]-3-(trimethylsilyl)-prop-2-yn-1-ols and 3phenyl-1-[(1-trimethylsilylethynyl)naphthalen-2-yl]prop-2-yn-1-ol. To a stirred solution of the alcohol in $\mathrm{Cl}_{2} \mathrm{CH}_{2}(c=0.1-1 \mathrm{M})$ at $\mathrm{rt}$ was added $\mathrm{MnO}_{2}$ in small portions until disappearance of starting material (typically 30-60 min, TLC monitoring, EtOAc/hexanes 1:9). The mixture was passed through a pad of silica to remove the solids and concentrated (purification by chromatography gives partial desilylation). Usually, the resulting ketones 12a-c and $\mathbf{1 7}$ are almost pure and no further purification is needed.

1-[1-(Phenylethynyl)naphthalen-2-yl]-3-(trimethylsilyl)propynone 12a<smiles>CC#CC(=O)c1ccc2ccccc2c1C#Cc1ccccc1</smiles>

Yellow oil; ${ }^{1} \mathrm{H}$ NMR $\left(\mathrm{CDCl}_{3}\right) \delta: 8.65(\mathrm{~d}, J=7.3 \mathrm{~Hz}, 1 \mathrm{H}, \mathrm{Ar} H), 8.10(\mathrm{~d}, J=7.5 \mathrm{~Hz}, 1 \mathrm{H}$, $\operatorname{Ar} H)$, 7.85-7.57 (m, 7H, $\operatorname{Ar} H)$, 7.42-7.37 (m, 2H, $\operatorname{Ar} H), 0.25\left(\mathrm{~s}, 9 \mathrm{H}, \mathrm{Si}\left(\mathrm{CH}_{3}\right)_{3}\right) ;{ }^{13} \mathrm{C}$ NMR/DEPT $\left(\mathrm{CDCl}_{3}\right) \delta: 177.7(C \mathrm{O}), 136.0(C), 134.7(C), 133.2(C), 132.0(2 \times C H), 128.9$ $(\mathrm{CH}), 128.7(\mathrm{CH}), 128.4(2 \times \mathrm{CH}), 128.1(2 \times \mathrm{CH}), 127.8(\mathrm{CH}), 127.6(\mathrm{CH}), 126.4(\mathrm{CH})$, 126.1 (C), $121.9(C), 102.3(C), 102.0(C), 101.1(C), 82.0(C),-0.8\left(\mathrm{Si}\left(\mathrm{CH}_{3}\right)_{3}\right)$; MS (70 eV) $\mathrm{m} / \mathrm{z}(\%)$ : $352\left(\mathrm{M}^{+}, 72\right), 337$ (100), 321 (68); HRMS $\left(\mathrm{C}_{24} \mathrm{H}_{20} \mathrm{OSi}\right)$ : calc. 352.1283; found, 352.1274 .

3-Phenyl-1-[(1-trimethylsilylethynyl)naphthalen-2-yl]propynone 17<smiles>CC#Cc1c(C(=O)C#Cc2ccccc2)ccc2ccccc12</smiles>

Clear oil; ${ }^{1} \mathrm{H}$ NMR $\left(\mathrm{CDCl}_{3}\right) \delta: 8.60(\mathrm{~d}, J=8.7 \mathrm{~Hz}, 1 \mathrm{H}, \mathrm{Ar} H), 8.09(\mathrm{~d}, J=8.7 \mathrm{~Hz}, 1 \mathrm{H}$, $\operatorname{ArH}$ ), 7.91-7.88 (m, 2H, $\operatorname{ArH}$ ), 7.70-7.64 (m, 4H, $\operatorname{ArH}$ ), 7.49-7.39 (m, 3H, $\operatorname{Ar} H$ ), 0.27 (s, 9H, $\left.\mathrm{Si}\left(\mathrm{CH}_{3}\right)_{3}\right) ;{ }^{13} \mathrm{C}$ NMR/DEPT $\left(\mathrm{CDCl}_{3}\right) \delta: 178.3(\mathrm{CO}), 137.5(\mathrm{C}), 134.6(\mathrm{C}), 133.6(\mathrm{C})$, $133.2(2 \times \mathrm{CH}), 130.7(\mathrm{CH}), 128.6(2 \times \mathrm{CH}), 128.5(\mathrm{CH}), 128.1(\mathrm{CH}), 127.8(\mathrm{CH}), 127.7$ $(\mathrm{CH}), 125.8(\mathrm{CH}), 121.5(C), 120.3(C), 108.9(C), 100.2(C), 93.9(C), 88.7(C),-0.2$ $\left(\mathrm{Si}\left(\mathrm{CH}_{3}\right)_{3}\right)$; MS (70 eV) m/z (\%): $352\left(\mathrm{M}^{+}, 73\right), 337$ (100), $278(83)$; HRMS $\left(\mathrm{C}_{24} \mathrm{H}_{20} \mathrm{OSi}\right)$ : calc. 352.1283; found, 352.1273 .

Thermal cyclizations: General procedure

A toluene or toluene/ $\mathrm{Et}_{3} \mathrm{~N}(8: 0.5)$ solution of the substrate $(c=20-65 \mathrm{mM})$ was placed in a sealed tube and heated overnight at $150{ }^{\circ} \mathrm{C}$ in a silicon oil bath. After evaporation of the 
solvent, the crude material was purified by column chromatography on silica gel using a mixture of hexanes/EtOAc (typically, 6:1) as eluent.

8-Methylbenzo[b]fluoren-11-one $\mathbf{3 b}$

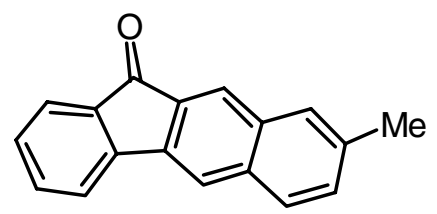

Yellow prisms, mp 144-146 ${ }^{\circ} \mathrm{C} ;{ }^{1} \mathrm{H}$ NMR $\left(\mathrm{CDCl}_{3}\right) \delta: 8.08(\mathrm{~s}, 1 \mathrm{H}, \mathrm{Ar} H), 7.82(\mathrm{~s}, 1 \mathrm{H}, \operatorname{Ar} H)$, 7.76-7.73 (m, 4H, $\operatorname{Ar} H), 7.55(\mathrm{td}, J=7.5,1.3 \mathrm{~Hz}, 1 \mathrm{H}, \operatorname{Ar} H), 7.38(\mathrm{dd}, J=8.3,1.6 \mathrm{~Hz}, 1 \mathrm{H}$, $\operatorname{Ar} H), 7.32(\operatorname{td}, J=7.6,1.0 \mathrm{~Hz}, 1 \mathrm{H}, \operatorname{Ar} H), 2.50\left(\mathrm{~s}, 3 \mathrm{H}, \mathrm{CH}_{3}\right) ;{ }^{13} \mathrm{C}$ NMR/DEPT $\left(\mathrm{CDCl}_{3}\right) \delta$ : $193.2(C \mathrm{C}), 144.9(C), 137.5(C), 136.8(C), 136.0(C), 134.9(C), 134.9(C H), 133.7(C)$, $132.7(\mathrm{C}), 131.0(\mathrm{CH}), 129.9(\mathrm{CH}), 128.8(\mathrm{CH}), 128.5(\mathrm{CH}), 125.0(\mathrm{CH}), 124.3(\mathrm{CH})$, $120.7(\mathrm{CH}), 118.8(\mathrm{CH}), 21.5\left(\mathrm{CH}_{3}\right)$; $\mathrm{MS}(70 \mathrm{eV}) \mathrm{m} / \mathrm{z}(\%): 244\left(\mathrm{M}^{+}, 100\right), 215(41)$.

8-Methoxybenzo[b]fluoren-11-one 3c

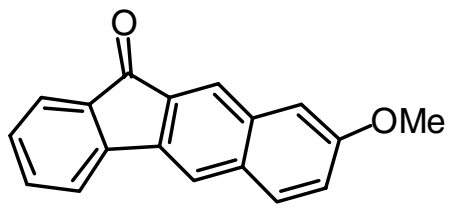

Yellow prisms; mp 203-204 ${ }^{\circ} \mathrm{C} ;{ }^{1} \mathrm{H}$ NMR $\left(\mathrm{CDCl}_{3}\right) \delta: 8.01(\mathrm{~s}, 1 \mathrm{H}, \operatorname{Ar} H), 7.74(\mathrm{~s}, 1 \mathrm{H}, \operatorname{Ar} H)$, 7.72-7.65 (m, 2H, $\operatorname{Ar} H), 7.61(\mathrm{~d}, J=7.6 \mathrm{~Hz}, 1 \mathrm{H}, \operatorname{Ar} H), 7.51$ (td, $J=7.6,0.8 \mathrm{~Hz}, 1 \mathrm{H}, \operatorname{Ar} H)$, $7.28(\mathrm{td}, J=7.6,0.8 \mathrm{~Hz}, 1 \mathrm{H}, \operatorname{ArH}), 7.20-7.14(\mathrm{~m}, 2 \mathrm{H}, \operatorname{Ar} H), 3.91\left(\mathrm{~s}, 3 \mathrm{H}, \mathrm{OCH}_{3}\right) ;{ }^{13} \mathrm{C}$

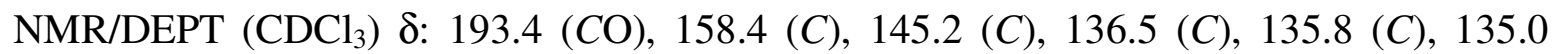
$(\mathrm{CH}), 134.9(C), 133.2(C), 132.0(C), 130.0(C \mathrm{H}), 128.7(\mathrm{CH}), 124.5(\mathrm{CH}), 124.4(\mathrm{CH})$, 121.1 $(\mathrm{CH}), 120.6(\mathrm{CH}), 118.9(\mathrm{CH}), 109.2(\mathrm{CH}), 55.4\left(\mathrm{CH}_{3}\right) ; \mathrm{MS}(70 \mathrm{eV}) \mathrm{m} / z(\%): 260$ $\left(\mathrm{M}^{+}, 100\right), 245$ (29), 217 (94), 189 (40); HRMS $\left(\mathrm{C}_{18} \mathrm{H}_{12} \mathrm{O}_{2}\right)$ : calc. 260.0837; found, 260.0838 .

8-Hydroxy-10-(trimethylsilyl)benzo[b]fluoren-11-one 3'd

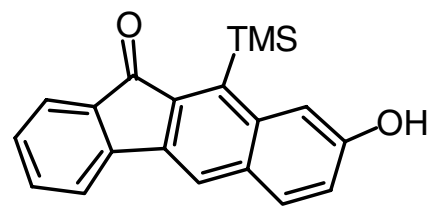

Yellow foam; ${ }^{1} \mathrm{H}$ NMR $\left(\mathrm{CDCl}_{3}\right) \delta: 7.80(\mathrm{~m}, 2 \mathrm{H}, \mathrm{Ar} H), 7.72-7.66(\mathrm{~m}, 2 \mathrm{H}, \mathrm{Ar} H), 7.63(\mathrm{~d}, J=$ $7.8 \mathrm{~Hz}, 1 \mathrm{H}, \operatorname{Ar} H), 7.51(\mathrm{td}, J=7.2,1.2 \mathrm{~Hz}, 1 \mathrm{H}, \mathrm{Ar} H), 7.30(\mathrm{dd}, J=7.2,0.9 \mathrm{~Hz}, 1 \mathrm{H}, \operatorname{Ar} H)$, $7.14(\mathrm{dd}, J=8.8,2.2 \mathrm{~Hz}, 1 \mathrm{H}, \operatorname{Ar} H), 5.59(\mathrm{~s}, 1 \mathrm{H}, \mathrm{OH}), 0.57\left(\mathrm{~s}, 9 \mathrm{H}, \mathrm{Si}\left(\mathrm{CH}_{3}\right)_{3}\right) ;{ }^{13} \mathrm{C}$ NMR/DEPT $\left(\mathrm{CDCl}_{3}\right)$ 8: $194.6(C \mathrm{CO}), 153.7(C), 144.5(C), 142.3(C), 139.6(C), 137.4(C)$, $136.4(C), 135.2(C), 134.7(C H), 130.9(C H), 130.7(C), 128.5(C H), 124.0(C H), 120.4$ $(\mathrm{CH}), 120.0(\mathrm{CH}), 119.1(\mathrm{CH}), 114.3(\mathrm{CH}), 3.1\left(\mathrm{Si}\left(\mathrm{CH}_{3}\right)_{3}\right) ; \mathrm{MS}(70 \mathrm{eV}) \mathrm{m} / z(\%): 318\left(\mathrm{M}^{+}\right.$, 10), 303 (100), 273 (15), $\mathrm{HRMS}\left(\mathrm{C}_{20} \mathrm{H}_{18} \mathrm{O}_{2} \mathrm{Si}\right)$ : calc. 318.1076; found, 318.1071. 
8-Nitro-10-(trimethylsilyl)benzo[b]fluoren-11-one 3'e

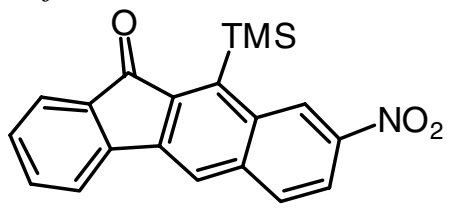

Yellow prisms; $\mathrm{mp}>280{ }^{\circ} \mathrm{C} ;{ }^{1} \mathrm{H}$ NMR $\left(\mathrm{THF}-d_{8}\right) \delta: 9.59(\mathrm{~d}, J=1.7 \mathrm{~Hz}, 1 \mathrm{H}, \mathrm{ArH}), 8.44-8.39$ $(\mathrm{m}, 2 \mathrm{H}, \operatorname{Ar} H), 8.20(\mathrm{~d}, J=8.9 \mathrm{~Hz}, 1 \mathrm{H}, \operatorname{Ar} H), 8.03(\mathrm{~d}, J=7.5 \mathrm{~Hz}, 1 \mathrm{H}, \operatorname{Ar} H), 7.87-7.75(\mathrm{~m}$, $2 \mathrm{H}, \mathrm{ArH}), 7.58(\mathrm{td}, J=7.4 \mathrm{~Hz}, 1 \mathrm{H}, \mathrm{ArH}), 0.77\left(\mathrm{~s}, 9 \mathrm{H}, \mathrm{Si}\left(\mathrm{CH}_{3}\right)_{3}\right)$; $\mathrm{MS}(70 \mathrm{eV}) \mathrm{m} / z(\%): 347$ $\left(\mathrm{M}^{+}, 4\right), 332$ (100), $286(24)$.

7-Methylbenzo[b]fluoren-11-one 3f

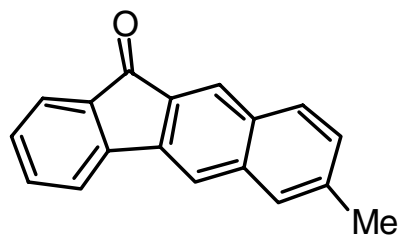

Yellow prisms; mp 150-152 ${ }^{\circ} \mathrm{C} ;{ }^{1} \mathrm{H}$ NMR $\left(\mathrm{CDCl}_{3}\right) \delta: 8.11(\mathrm{~s}, 1 \mathrm{H}, \mathrm{ArH}), 7.88-7.65(\mathrm{~m}, 4 \mathrm{H}$, $\operatorname{Ar} H), 7.58(\mathrm{~s}, 1 \mathrm{H}, \operatorname{Ar} H), 7.54(\mathrm{td}, J=7.5,0.9 \mathrm{~Hz}, 1 \mathrm{H}, \operatorname{Ar} H), 7.36-7.26(\mathrm{~m}, 2 \mathrm{H}, \operatorname{Ar} H), 2.51$ $\left(\mathrm{s}, 3 \mathrm{H}, \mathrm{CH}_{3}\right) ;{ }^{13} \mathrm{C} \mathrm{NMR} / \mathrm{DEPT}\left(\mathrm{CDCl}_{3}\right)$ 8: $193.0(\mathrm{CO}), 144.7(\mathrm{C}), 139.2(\mathrm{C}), 138.3(\mathrm{C})$, $137.0(C), 136.1(C), 134.7(C H), 131.9(C), 131.6(C), 130.4(C H), 128.9(2 \times C H), 128.0$ $(\mathrm{CH}), 125.3(\mathrm{CH}), 124.2(\mathrm{CH}), 120.8(\mathrm{CH}), 118.3(\mathrm{CH}), 21.8\left(\mathrm{CH}_{3}\right)$; MS $(70 \mathrm{eV}) \mathrm{m} / \mathrm{z}(\%)$ : $244\left(\mathrm{M}^{+}, 100\right), 215(56)$.

7-Methoxybenzo[b]fluoren-11-one $\mathbf{3 g}$

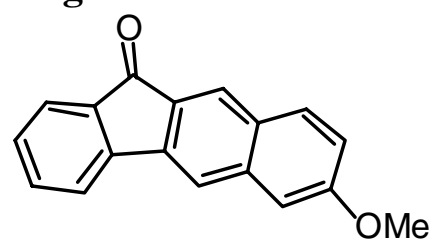

Yellow prisms; mp $163-164{ }^{\circ} \mathrm{C} ;{ }^{1} \mathrm{H}$ NMR $\left(\mathrm{CDCl}_{3}\right) \delta: 8.10(\mathrm{~s}, 1 \mathrm{H}, \mathrm{Ar} H), 7.81-7.66(\mathrm{~m}, 4 \mathrm{H}$, $\operatorname{Ar} H), 7.55(\mathrm{td}, J=7.5,1.0 \mathrm{~Hz}, 1 \mathrm{H}, \operatorname{Ar} H), 7.34(\mathrm{td}, J=7.5,1.0 \mathrm{~Hz}, 1 \mathrm{H}, \operatorname{Ar} H), 7.16(\mathrm{~d}, J=$ 2.5, $1.0 \mathrm{~Hz}, 1 \mathrm{H}, \mathrm{ArH}), 7.11(\mathrm{dd}, J=8.8,1.6 \mathrm{~Hz}, 1 \mathrm{H}, \mathrm{ArH}), 3.95\left(\mathrm{~s}, 3 \mathrm{H}, \mathrm{OCH}_{3}\right) ;{ }^{13} \mathrm{C}$ NMR/DEPT $\left(\mathrm{CDCl}_{3}\right)$ $\delta: 192.9(C O), 160.1(C), 144.5(C), 139.2(C), 138.6(C), 136.2(C)$, $134.6(C H), 132.1(C H), 130.7(C), 129.0(C H), 128.5(C), 125.4(C H), 124.1(C H), 120.7$ $(C \mathrm{H}), 118.8(\mathrm{CH}), 117.9(\mathrm{CH}), 107.7(\mathrm{CH}), 55.3\left(\mathrm{CH}_{3}\right)$; MS $(70 \mathrm{eV}) \mathrm{m} / z(\%): 260\left(\mathrm{M}^{+}\right.$, 100), 217 (76), 189 (44).

9-Methoxybenzo[b]fluoren-11-one 3g' (partially described, ref. 4)<smiles>COc1cccc2cc3c(cc12)C(=O)c1ccccc1-3</smiles> 
Yellow prisms; mp $184-186{ }^{\circ} \mathrm{C} ;{ }^{1} \mathrm{H}$ NMR $\left(\mathrm{CDCl}_{3}\right) \delta: 8.65(\mathrm{~s}, 1 \mathrm{H}, \mathrm{Ar} H), 7.81(\mathrm{~s}, 1 \mathrm{H}, \mathrm{Ar} H)$, 7.78-7.67 (m, 2H, ArH), $7.55(\mathrm{td}, J=7.5,1.2 \mathrm{~Hz}, 1 \mathrm{H}, \operatorname{ArH}), 7.50-7.30(\mathrm{~m}, 3 \mathrm{H}, \operatorname{ArH}), 6.81$ $(\mathrm{dd}, J=7.5,1.2 \mathrm{~Hz}, 1 \mathrm{H}, \mathrm{ArH}), 4.00\left(\mathrm{~s}, 3 \mathrm{H}, \mathrm{OCH}_{3}\right) ;{ }^{13} \mathrm{C} \mathrm{NMR} / \mathrm{DEPT}\left(\mathrm{CDCl}_{3}\right) \delta: 193.2$ (CO), $157.8(C), 144.6(C), 139.0(C), 138.0(C), 136.3(C), 134.8(C H), 131.8(C), 129.6$ $(C H), 129.1(C H), 125.4(C), 124.4(C H), 120.9(C H), 120.9(C H), 120.6(C H), 118.6$ $(C \mathrm{H}), 105.5(\mathrm{CH}), 55.6\left(\mathrm{CH}_{3}\right)$; MS (70 eV) m/z (\%): $260\left(\mathrm{M}^{+}, 100\right), 217(90), 189(36)$.

7-Hydroxy-10-(trimethylsilyl)benzo[b]fluoren-11-one 3'h

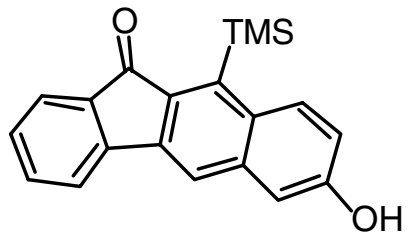

Yellow crystals; ${ }^{1} \mathrm{H}$ NMR (acetone- $d_{6}$ ) $\delta: 9.09$ (s, $\left.1 \mathrm{H}, \mathrm{OH}\right), 8.30$ (d, J=9.1 Hz, 1H, ArH), 7.92 (s, 1H, ArH), 7.80 (d, J= $7.5 \mathrm{~Hz}, 1 \mathrm{H}, \operatorname{ArH}), 7.62$ (d, J= $7.5 \mathrm{~Hz}, 1 \mathrm{H}, \operatorname{ArH}), 7.55$ (t, J= $7.5 \mathrm{~Hz}, 1 \mathrm{H}, \operatorname{ArH}), 7.35$ (t, $J=7.5 \mathrm{~Hz}, 1 \mathrm{H}, \operatorname{ArH}), 7.24$ (d, $J=2.5 \mathrm{~Hz}, 1 \mathrm{H}, \operatorname{ArH}), 7.13$ (dd, $J=$ 9.1, $2.8 \mathrm{~Hz}, 1 \mathrm{H}, \mathrm{ArH}), 0.55\left(\mathrm{~s}, 9 \mathrm{H}, \mathrm{Si}\left(\mathrm{CH}_{3}\right)_{3}\right) ;{ }^{13} \mathrm{C}$ NMR/DEPT (acetone- $\left.d_{6}\right) \delta: 195.0(\mathrm{CO})$, $158.8(C), 144.1(C), 145.0(C), 140.1(C), 139.7(C), 138.5(C), 137.1(C), 136.0(C H)$, $134.3(\mathrm{CH}), 133.9(C), 130.4(\mathrm{CH}), 124.7(C \mathrm{H}), 122.0(\mathrm{CH}), 120.9(C \mathrm{H}), 119.2(C \mathrm{H})$, $113.0(\mathrm{CH}), 3.5\left(\mathrm{Si}\left(\mathrm{CH}_{3}\right)_{3}\right)$; MS (70 eV) m/z (\%): $318\left(\mathrm{M}^{+}, 6\right), 303$ (100), 273 (14), 200 (5); HRMS $\left(\mathrm{C}_{20} \mathrm{H}_{18} \mathrm{O}_{2} \mathrm{Si}\right)$ : calc. 318.1076; found, 318.1068.

\section{7-Hydroxybenzo[b]fluoren-11-one $\mathbf{3 h}$}

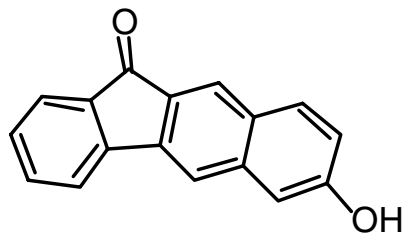

Yellow prisms; mp 169-170 ${ }^{\circ} \mathrm{C} ;{ }^{1} \mathrm{H} \mathrm{NMR}\left(\mathrm{CDCl}_{3}\right) \delta: 8.10(\mathrm{~s}, 1 \mathrm{H}, \mathrm{ArH}), 7.78(\mathrm{~d}, J=8.7 \mathrm{~Hz}$, 1H, ArH), $7.74(\mathrm{~d}, J=7.6 \mathrm{~Hz}, 1 \mathrm{H}, \operatorname{Ar} H), 7.71(\mathrm{~s}, 1 \mathrm{H}, \operatorname{Ar} H), 7.68$ (d, J=7.6 Hz, 1H, ArH), $7.54(\mathrm{t}, J=7.6 \mathrm{~Hz}, 1 \mathrm{H}, \operatorname{ArH}), 7.34(\mathrm{t}, J=7.3 \mathrm{~Hz}, 1 \mathrm{H}, \operatorname{Ar} H), 7.18(\mathrm{~d}, J=2.4 \mathrm{~Hz}, 1 \mathrm{H}, \operatorname{Ar} H)$, $7.07(\mathrm{dd}, J=8.5,2.4 \mathrm{~Hz}, 1 \mathrm{H}, \operatorname{Ar} H), 5.69(\mathrm{~s}, 1 \mathrm{H}, \mathrm{OH}) ;{ }^{13} \mathrm{C}$ NMR/DEPT (acetone- $\left.d_{6}\right) \delta$ : 193.1 (CO), $159.7(C), 144.8(C), 140.4(C), 140.2(C), 137.5(C), 136.2(C H), 133.9(C H)$, $131.4(C), 130.5(C H), 129.3(C), 126.5(C H), 124.9(C H), 122.5(C H), 120.1(C H), 119.2$ $(C \mathrm{H}), 112.3(\mathrm{CH})$; MS (70 eV) m/z (\%): $246\left(\mathrm{M}^{+}, 100\right), 189$ (32), 50 (48); HRMS $\left(\mathrm{C}_{17} \mathrm{H}_{10} \mathrm{O}\right)$ : calc. 246.0680 ; found, 246.0684 .

9-Hydroxybenzo[b]fluoren-11-one $\mathbf{3 h}$ '

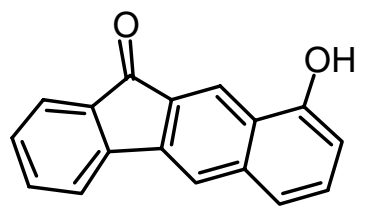


Yellow solid; ${ }^{1} \mathrm{H}$ NMR $\left(\mathrm{CDCl}_{3}\right) \delta: 9.45$ (broad s, $\left.1 \mathrm{H}, \mathrm{OH}\right), 8.55(\mathrm{~s}, 1 \mathrm{H}, \mathrm{ArH}), 8.10(\mathrm{~s}, 1 \mathrm{H}$, $\operatorname{Ar} H), 7.95(\mathrm{~d}, J=7.5 \mathrm{~Hz}, 1 \mathrm{H}, \operatorname{Ar} H), 7.73-7.64(\mathrm{~m}, 2 \mathrm{H}, \operatorname{Ar} H), 7.48-7.40$ (m, 3H, $\operatorname{Ar} H), 6.98$ $(\mathrm{t}, J=4.3 \mathrm{~Hz}, 1 \mathrm{H}, \mathrm{ArH})$; MS (70 eV) $m / z(\%): 246\left(\mathrm{M}^{+}, 100\right), 189(55)$.

6-Methylbenzo[b]fluoren-11-one 3i

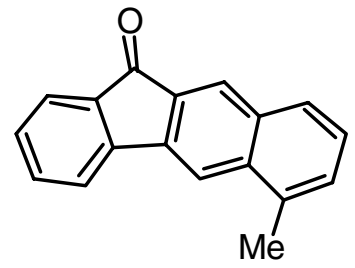

Yellow prisms; mp 154-155 ${ }^{\circ} \mathrm{C} ;{ }^{1} \mathrm{H}$ NMR $\left(\mathrm{CDCl}_{3}\right) \delta: 8.10(\mathrm{~s}, 1 \mathrm{H}, \mathrm{Ar} H), 7.95(\mathrm{~s}, 1 \mathrm{H}, \mathrm{Ar} H)$, 7.75-7.66 (m, 3H, ArH), $7.52(\mathrm{t}, J=7.3 \mathrm{~Hz}, 1 \mathrm{H}, \operatorname{ArH}), 7.39-7.22(\mathrm{~m}, 3 \mathrm{H}, \operatorname{ArH}), 2.67(\mathrm{~s}, 3 \mathrm{H}$, $\left.\mathrm{CH}_{3}\right) ;{ }^{13} \mathrm{C} \mathrm{NMR/DEPT}\left(\mathrm{CDCl}_{3}\right) \delta: 193.2(\mathrm{CO}), 145.0(\mathrm{C}), 138.2(\mathrm{C}), 136.1(\mathrm{C}), 135.9(\mathrm{C})$, $135.2(C), 134.9(C H), 133.7(C), 132.2(C), 130.0(C H), 129.2(C H), 129.1(C H), 126.6$ $(\mathrm{CH}), 126.1(\mathrm{CH}), 124.4(\mathrm{CH}), 120.8(\mathrm{CH}), 115.3(\mathrm{CH}), 19.6\left(\mathrm{CH}_{3}\right) ; \mathrm{MS}(70 \mathrm{eV}) \mathrm{m} / \mathrm{z}(\%)$ : $244\left(\mathrm{M}^{+}, 100\right), 243(25), 215$ (46).

6-Methoxybenzo[b]fluoren-11-one $\mathbf{3 j}$

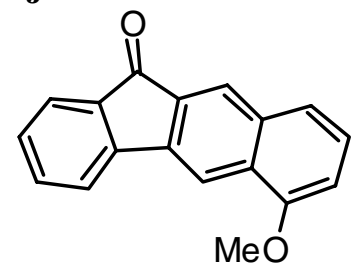

Yellow prisms; mp 154-156 ${ }^{\circ} \mathrm{C} ;{ }^{1} \mathrm{H}$ NMR $\left(\mathrm{CDCl}_{3}\right) \delta: 8.32(\mathrm{~s}, 1 \mathrm{H}, \mathrm{Ar} H), 8.12(\mathrm{~s}, 1 \mathrm{H}, \operatorname{Ar} H)$, 7.77-7.71 (m, 2H, ArH), 7.55 (td, J=7.2, $1.5 \mathrm{~Hz}, 1 \mathrm{H}, \operatorname{Ar} H), 7.48(\mathrm{~d}, J=7.4 \mathrm{~Hz}, 1 \mathrm{H}, \operatorname{Ar} H)$, 7.40 (d, J=7.9 Hz, 1H, ArH), 7.36-7.29 (m, 1H, ArH), 6.92 (dd, J= 7.5, 1.1 Hz, 1H, ArH), $4.04\left(\mathrm{~s}, 3 \mathrm{H}, \mathrm{OCH}_{3}\right) ;{ }^{13} \mathrm{C} \mathrm{NMR} / \mathrm{DEPT}\left(\mathrm{CDCl}_{3}\right) \delta$ : $193.3(\mathrm{CO}), 155.9(\mathrm{C}), 145.2(\mathrm{C}), 137.6$ $(C), 135.9(C), 135.0(C H), 134.5(C), 133.0(C), 128.9(C H), 128.4(C), 127.0(C H), 125.1$ $(\mathrm{CH}), 124.3(\mathrm{CH}), 122.9(\mathrm{CH}), 121.0(\mathrm{CH}), 113.5(\mathrm{CH}), 107.2(\mathrm{CH}), 55.6\left(\mathrm{CH}_{3}\right)$; MS (70 eV) $m / z(\%): 260\left(\mathrm{M}^{+}, 100\right), 245$ (62), 217 (76).

6-t-Butyl-10-(trimethylsilyl)benzo[b]fluoren-11-one 3'k

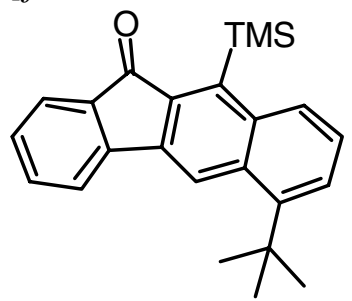

Yellow prisms; mp 170-171 ${ }^{\circ} \mathrm{C} ;{ }^{1} \mathrm{H}$ NMR $\left(\mathrm{CDCl}_{3}\right) \delta: 8.57(\mathrm{~s}, 1 \mathrm{H}, \mathrm{ArH}), 8.26(\mathrm{~d}, J=8.5 \mathrm{~Hz}$, 1H, $\operatorname{Ar} H), 7.75-7.69(\mathrm{~m}, 2 \mathrm{H}, \operatorname{Ar} H), 7.57$ (d, J=7.5 Hz, 1H, $\operatorname{Ar} H), 7.53$ (d, J= 8.5 Hz, 1H, 
$\operatorname{ArH}), 7.36(\mathrm{t}, J=7.5 \mathrm{~Hz}, 2 \mathrm{H}, \mathrm{ArH}), 1.68\left(\mathrm{~s}, 9 \mathrm{H}, \mathrm{C}\left(\mathrm{CH}_{3}\right)_{3}\right), 0.57\left(\mathrm{~s}, 9 \mathrm{H}, \mathrm{Si}\left(\mathrm{CH}_{3}\right)_{3}\right) ;{ }^{13} \mathrm{C}$ NMR/DEPT $\left(\mathrm{CDCl}_{3}\right)$ 8: $195.0(C O), 147.7(C), 146.7(C), 145.1(C), 140.5(C), 139.2(C)$, $137.1(C), 136.2(C), 135.1(C H), 134.4(C), 130.9(C H), 129.3(C H), 126.3(C H), 125.6$ $(\mathrm{CH}), 124.5(\mathrm{CH}), 120.5(\mathrm{CH}), 119.3(\mathrm{CH}), 36.6(\mathrm{C}), 32.7\left(\mathrm{C}\left(\mathrm{CH}_{3}\right)_{3}\right), 3.4\left(\mathrm{Si}\left(\mathrm{CH}_{3}\right)_{3}\right)$; $\mathrm{MS}$ $(70 \mathrm{eV}) \mathrm{m} / \mathrm{z}(\%): 358\left(\mathrm{M}^{+}, 10\right), 343(100), 327(20), 313(9)$.

8-Methoxy-6-methyl-10-(trimethylsilyl)benzo[b]fluoren-11-one 3'm

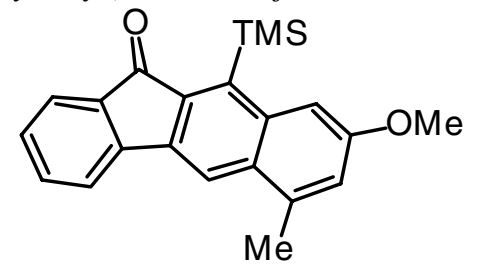

Yellow prisms; mp 138-141 ${ }^{\circ} \mathrm{C} ;{ }^{1} \mathrm{H}$ NMR $\left(\mathrm{CDCl}_{3}\right)$ 8: $7.93(\mathrm{~s}, 1 \mathrm{H}, \mathrm{ArH}), 7.65-7.62(\mathrm{~m}, 3 \mathrm{H}$, $\operatorname{Ar} H$ ), $7.48(\mathrm{t}, J=7.3 \mathrm{~Hz}, 1 \mathrm{H}, \operatorname{Ar} H), 7.25(\mathrm{t}, J=7.3 \mathrm{~Hz}, 1 \mathrm{H}, \operatorname{Ar} H), 7.02(\mathrm{~s}, 1 \mathrm{H}, \operatorname{Ar} H), 3.89$

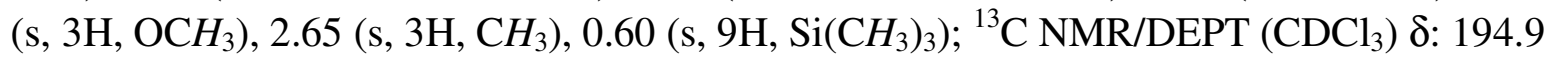
(CO), $157.2(C), 144.7(C), 143.0(C), 140.0(C), 136.8(C), 136.7(C), 135.2(C), 131.4$ $(C), 134.7(C H), 130.0(C), 128.4(C H), 123.9(C H), 121.1(C H), 119.9(C H), 116.4(C H)$, $109.0(\mathrm{CH}), 55.3\left(\mathrm{CH}_{3}\right), 20.0\left(\mathrm{CH}_{3}\right), 3.0\left(\mathrm{Si}\left(\mathrm{CH}_{3}\right)_{3}\right)$; $\mathrm{MS}(70 \mathrm{eV}) \mathrm{m} / z(\%): 346\left(\mathrm{M}^{+}, 18\right)$, 332 (29), 331 (100), 287 (24); HRMS $\left(\mathrm{C}_{22} \mathrm{H}_{22} \mathrm{O}_{2} \mathrm{Si}\right)$ : calc. 346.1389; found, 346.1389.

8-Methoxy-6-methylbenzo[b]fluoren-11-one 3m

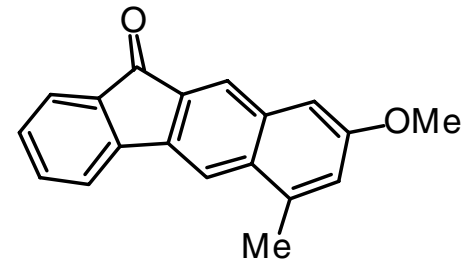

Yellow crystals; mp $155-157{ }^{\circ} \mathrm{C} ;{ }^{1} \mathrm{H}$ NMR $\left(\mathrm{CDCl}_{3}\right) \delta$ : 7.93 (broad s, $1 \mathrm{H}, \mathrm{Ar} H$ ), 7.8 (broad s, $1 \mathrm{H}, \operatorname{Ar} H), 7.66(\mathrm{~d}, J=7.4 \mathrm{~Hz}, 1 \mathrm{H}, \operatorname{Ar} H), 7.60(\mathrm{dd}, J=7.4,2.3 \mathrm{~Hz}, 1 \mathrm{H}, \operatorname{Ar} H), 7.48(\mathrm{t}, J=$ $7.4 \mathrm{~Hz}, 1 \mathrm{H}, \operatorname{Ar} H$ ), 7.26 (t, J= $7.4 \mathrm{~Hz}, 1 \mathrm{H}, \operatorname{Ar} H$ ), 6.98 (broad s, 2H, $\operatorname{Ar} H$ ), 3.87 (s, 3H, $\left.\mathrm{OCH}_{3}\right), 2.61\left(\mathrm{~s}, 3 \mathrm{H}, \mathrm{CH}_{3}\right) ;{ }^{13} \mathrm{C}$ NMR/DEPT $\left(\mathrm{CDCl}_{3}\right) \delta: 193.4(\mathrm{CO}), 157.8(\mathrm{C}), 145.3(\mathrm{C})$, $136.8(C), 136.3(C), 135.7(C), 135.3(C), 134.8(C H), 132.8(C), 131.3(C), 128.5(C H)$, $124.9(\mathrm{CH}), 124.2(\mathrm{CH}), 121.9(\mathrm{CH}), 120.4(\mathrm{CH}), 115.2(\mathrm{CH}), 107.4(\mathrm{CH}), 55.2\left(\mathrm{CH}_{3}\right)$, $19.4\left(\mathrm{CH}_{3}\right) ; \mathrm{MS}(70 \mathrm{eV}) \mathrm{m} / \mathrm{z}(\%): 274\left(\mathrm{M}^{+}, 100\right), 231$ (29); HRMS $\left(\mathrm{C}_{19} \mathrm{H}_{14} \mathrm{O}_{2}\right)$ : calc. 274.0993; found, 274.0996.

6,8-Dimethoxy-10-(trimethylsilyl)benzo[b]fluoren-11-one 3'n<smiles>COc1cc(OC)c2cc3c(cc2c1C)C(=O)c1ccccc1C3=O</smiles> 
Yellow crystals; mp 159-161 ${ }^{\circ} \mathrm{C} ;{ }^{1} \mathrm{H}$ NMR $\left(\mathrm{CDCl}_{3}\right) \delta: 8.30(\mathrm{~s}, 1 \mathrm{H}, \mathrm{Ar} H), 7.69-7.64$ (m, 2H, $\operatorname{Ar} H), 7.50(\mathrm{td}, J=7.3,1.1 \mathrm{~Hz}, 1 \mathrm{H}, \operatorname{ArH}), 7.38(\mathrm{~d}, J=1.2 \mathrm{~Hz}, 1 \mathrm{H}, \operatorname{ArH}), 7.27(\mathrm{td}, J=7.3,1.1$ $\mathrm{Hz}, 1 \mathrm{H}, \mathrm{ArH}), 6.59$ (d, J=2.2 Hz, 1H, $\mathrm{ArH}), 4.00$ (s, 3H, OCH $\mathrm{OH}_{3}, 3.92$ (s, 3H, OCH $), 0.59$ $\left(\mathrm{s}, 9 \mathrm{H}, \mathrm{Si}\left(\mathrm{CH}_{3}\right)_{3}\right) ;{ }^{13} \mathrm{C}$ NMR/DEPT $\left(\mathrm{CDCl}_{3}\right) \delta: 195.6(\mathrm{CO}), 158.8(C), 157.3(C), 145.4(C)$, 142.2 (C), $141.1(C), 140.6(C), 136.6(C), 135.5(C), 135.2(C H), 128.7(C H), 124.3(C H)$, $123.5(C), 120.5(C H), 115.2(C H), 103.3(C H), 100.1(C H), 56.1\left(C_{3}\right), 55.9\left(C_{3}\right), 3.4$ $\left(\mathrm{Si}\left(\mathrm{CH}_{3}\right)_{3}\right)$; MS (70 eV) m/z (\%): $362\left(\mathrm{M}^{+}, 23\right), 348$ (28), 347 (100), 289 (23), 261 (9); HRMS $\left(\mathrm{C}_{22} \mathrm{H}_{22} \mathrm{O}_{3} \mathrm{Si}\right)$ : calc. 362.1338; found, 362.1323.

6,8-Dimethyl-10-(trimethylsilyl)benzo[b]fluoren-11-one 3'o

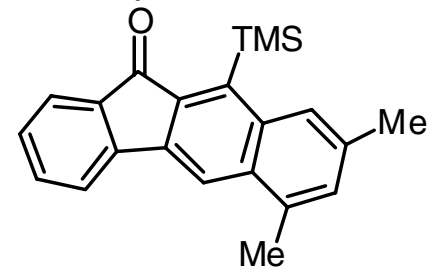

Yellow prisms; mp 146-148 ${ }^{\circ} \mathrm{C} ;{ }^{1} \mathrm{H}$ NMR $\left(\mathrm{CDCl}_{3}\right) \delta: 8.06($ broad s, $1 \mathrm{H}, \mathrm{ArH}), 8.03(\mathrm{~s}, 1 \mathrm{H}$, $\operatorname{Ar} H), 7.71(\mathrm{~d}, J=7.6 \mathrm{~Hz}, 1 \mathrm{H}, \operatorname{ArH}), 7.68(\mathrm{~d}, J=7.1 \mathrm{~Hz}, 1 \mathrm{H}, \operatorname{Ar} H), 7.53(\mathrm{t}, J=7.1 \mathrm{~Hz}, 1 \mathrm{H}$, $\mathrm{ArH}), 7.31$ (t, J=7.6 Hz, 1H, ArH), 7.22 (broad s, 1H, ArH), $2.70\left(\mathrm{~s}, 3 \mathrm{H}, \mathrm{CH}_{3}\right), 2.46$ (s, 3H, $\left.\mathrm{CH}_{3}\right), 0.58$ (s, 9H, $\left.\mathrm{Si}\left(\mathrm{CH}_{3}\right)_{3}\right)$; ${ }^{13} \mathrm{C}$ NMR/DEPT $\left(\mathrm{CDCl}_{3}\right) \delta: 194.8(\mathrm{CO}), 144.6(\mathrm{C}), 144.4$ $(C), 139.6(C), 138.8(C), 137.5(C), 135.6(C), 135.2(C), 135.0(C), 134.6(C H), 132.7$ $(C), 131.4(C H), 129.2(C H), 128.6(C H), 123.9(C H), 120.1(C H), 116.3(C H), 21.7\left(C_{3}\right)$, $19.9\left(\mathrm{CH}_{3}\right), 3.0\left(\mathrm{Si}\left(\mathrm{CH}_{3}\right)_{3}\right)$; $\mathrm{MS}(70 \mathrm{eV}) \mathrm{m} / z(\%): 330\left(\mathrm{M}^{+}, 10\right), 315$ (100), $285(14)$.

\section{6,8-Dimethylbenzo[b]fluoren-11-one 30}

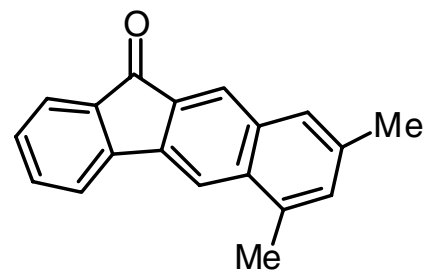

Yellow prisms; mp 185-186 ${ }^{\circ} \mathrm{C} ;{ }^{1} \mathrm{H}$ NMR $\left(\mathrm{CDCl}_{3}\right) \delta: 7.98(\mathrm{~s}, 1 \mathrm{H}, \mathrm{Ar} H), 7.86(\mathrm{~s}, 1 \mathrm{H}, \mathrm{Ar} H)$, 7.72-7.62 (m, 2H, ArH), $7.50(\mathrm{t}, J=7.4 \mathrm{~Hz}, 1 \mathrm{H}, \mathrm{ArH}), 7.44($ broad s, 1H, ArH), $7.28(\mathrm{t}, J=$ $7.4 \mathrm{~Hz}, 1 \mathrm{H}, \mathrm{ArH}), 7.17$ (broad s, $1 \mathrm{H}, \mathrm{ArH}), 2.63\left(\mathrm{~s}, 3 \mathrm{H}, \mathrm{CH}_{3}\right), 2.42\left(\mathrm{~s}, 3 \mathrm{H}, \mathrm{CH}_{3}\right) ;{ }^{13} \mathrm{C}$ NMR/DEPT $\left(\mathrm{CDCl}_{3}\right)$ \&: $193.4(C \mathrm{O}), 145.2(C), 137.4(C), 136.4(C), 136.0(C), 134.9(C)$, $134.8(\mathrm{CH}), 134.0(C), 134.0(C), 132.3(C), 132.2(C H), 128.8(C H), 128.3(C H), 125.5$ $(\mathrm{CH}), 124.3(\mathrm{CH}), 120.6(\mathrm{CH}), 115.1(\mathrm{CH}), 21.4\left(\mathrm{CH}_{3}\right), 19.4\left(\mathrm{CH}_{3}\right) ; \mathrm{MS}(70 \mathrm{eV}) \mathrm{m} / z(\%)$ : $258\left(\mathrm{M}^{+}, 100\right), 243(28)$.

4-Methyl-6-(trimethylsilyl)benzo[c]fluoren-7-one 7b 


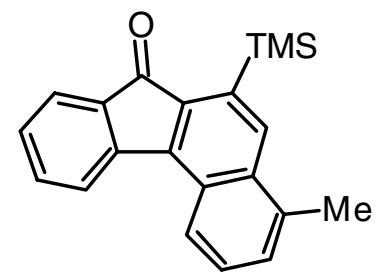

Orange prisms; mp $165-167{ }^{\circ} \mathrm{C} ;{ }^{1} \mathrm{H}$ NMR $\left(\mathrm{CDCl}_{3}\right) \delta: 8.40(\mathrm{~d}, J=8.1 \mathrm{~Hz}, 1 \mathrm{H}, \operatorname{Ar} H), 8.16(\mathrm{~s}$, $1 \mathrm{H}, \operatorname{Ar} H), 8.05(\mathrm{~d}, J=7.6 \mathrm{~Hz}, 1 \mathrm{H}, \mathrm{Ar} H), 7.68-7.63(\mathrm{~m}, 1 \mathrm{H}, \operatorname{Ar} H), 7.56-7.40(\mathrm{~m}, 3 \mathrm{H}, \mathrm{Ar} H)$, 7.30 (t, $J=7.4 \mathrm{~Hz}, 1 \mathrm{H}, \mathrm{Ar} H), 2.74\left(\mathrm{~s}, 3 \mathrm{H}, \mathrm{CH}_{3}\right), 0.45$ (s, 9H, $\left.\mathrm{Si}\left(\mathrm{CH}_{3}\right)_{3}\right) ;{ }^{13} \mathrm{C}$ NMR/DEPT $\left(\mathrm{CDCl}_{3}\right)$ \&: $195.9(C \mathrm{C}), 145.2(C), 143.4(C), 136.0(C), 135.8(C), 135.6(C), 134.6(C)$, $134.3(\mathrm{CH}), 134.2(\mathrm{C}), 132.8(\mathrm{CH}), 129.4(\mathrm{C}), 129.2(\mathrm{CH}), 128.5(\mathrm{CH}), 127.7(\mathrm{CH}), 123.7$ $(\mathrm{CH}), 123.4(\mathrm{CH}), 122.8(\mathrm{CH}), 20.1\left(\mathrm{CH}_{3}\right),-0.9\left(\mathrm{Si}\left(\mathrm{CH}_{3}\right)_{3}\right)$; MS $(70 \mathrm{eV}) \mathrm{m} / z(\%): 316\left(\mathrm{M}^{+}\right.$, 10), 302 (33), 301 (100).

4-Methoxy-6-(trimethylsilyl)benzo[c]fluoren-7-one 7c

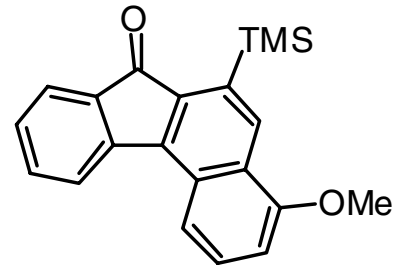

Orange crystals; mp 212-214 ${ }^{\circ} \mathrm{C} ;{ }^{1} \mathrm{H}$ NMR $\left(\mathrm{CDCl}_{3}\right) \delta: 8.45(\mathrm{~s}, 1 \mathrm{H}, \mathrm{Ar} H), 8.00(\mathrm{~d}, J=8.6 \mathrm{~Hz}$, $1 \mathrm{H}, \operatorname{Ar} H), 7.95(\mathrm{~d}, J=7.5 \mathrm{~Hz}, 1 \mathrm{H}, \operatorname{Ar} H), 7.62(\mathrm{~d}, J=7.0 \mathrm{~Hz}, 1 \mathrm{H}, \operatorname{Ar} H), 7.53-7.41(\mathrm{~m}, 2 \mathrm{H}$, $\mathrm{ArH}), 7.25$ (t, $J=7.0 \mathrm{~Hz}, 1 \mathrm{H}, \mathrm{ArH}), 6.87(\mathrm{~d}, J=7.7 \mathrm{~Hz}, 1 \mathrm{H}, \mathrm{ArH}), 4.01\left(\mathrm{~s}, 3 \mathrm{H}, \mathrm{OCH}_{3}\right), 0.45$ (s, 9H, $\left.\mathrm{Si}\left(\mathrm{CH}_{3}\right)_{3}\right) ;{ }^{13} \mathrm{C}$ NMR/DEPT $\left(\mathrm{CDCl}_{3}\right)$ 8: $196.0(\mathrm{CO}), 156.1(\mathrm{C}), 145.2(\mathrm{C}), 142.5(\mathrm{C})$, $136.3(C), 134.3(\mathrm{CH}), 134.0(C), 133.9(C), 130.5(\mathrm{CH}), 130.0(C), 128.6(C), 128.3(\mathrm{CH})$, $128.3(\mathrm{CH}), 123.6(\mathrm{CH}), 123.2(\mathrm{CH}), 116.6(\mathrm{CH}), 106.1(\mathrm{CH}), 46.8\left(\mathrm{CH}_{3}\right),-0.8\left(\mathrm{Si}\left(\mathrm{CH}_{3}\right)_{3}\right)$; MS (70 eV) m/z (\%): $332\left(\mathrm{M}^{+}, 11\right), 317$ (100), 274 (31); HRMS $\left(\mathrm{C}_{21} \mathrm{H}_{20} \mathrm{O}_{2} \mathrm{Si}\right)$ : calc. 332.1233; found, 332.1229 .

3-Methyl-6-(trimethylsilyl)benzo[c]fluoren-7-one 7f

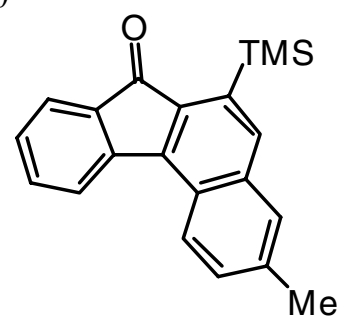

Orange prisms; mp 134-136 ${ }^{\circ} \mathrm{C} ;{ }^{1} \mathrm{H}$ NMR $\left(\mathrm{CDCl}_{3}\right) \delta: 8.39(\mathrm{~d}, J=8.5 \mathrm{~Hz}, 1 \mathrm{H}, \mathrm{ArH}), 8.01(\mathrm{~d}$, $J=7.9 \mathrm{~Hz}, 1 \mathrm{H}, \operatorname{Ar} H), 7.85(\mathrm{~s}, 1 \mathrm{H}, \operatorname{Ar} H), 7.69-7.62(\mathrm{~m}, 2 \mathrm{H}, \operatorname{Ar} H), 7.55-7.42(\mathrm{~m}, 2 \mathrm{H}, \operatorname{Ar} H)$, 7.34-7.25 (m, 1H, $\mathrm{ArH}), 2.54\left(\mathrm{~s}, 3 \mathrm{H}, \mathrm{CH}_{3}\right), 0.43\left(\mathrm{~s}, 9 \mathrm{H}, \mathrm{Si}\left(\mathrm{CH}_{3}\right)_{3}\right) ;{ }^{13} \mathrm{C}$ NMR/DEPT $\left(\mathrm{CDCl}_{3}\right)$ \&: $195.8(\mathrm{CO}), 145.0(C), 143.1(C), 138.5(C), 137.1(C), 136.3(\mathrm{CH}), 135.2(C)$, $135.1(C), 134.3(C), 134.2(C), 130.3(\mathrm{CH}), 128.7(\mathrm{CH}), 128.5(\mathrm{CH}), 127.3(\mathrm{CH}), 124.3$ 
$(\mathrm{CH}), 123.6(\mathrm{CH}), 123.1(\mathrm{CH}), 21.8\left(\mathrm{CH}_{3}\right),-0.9\left(\mathrm{Si}\left(\mathrm{CH}_{3}\right)_{3}\right) ; \mathrm{MS}(70 \mathrm{eV}) \mathrm{m} / z(\%): 316\left(\mathrm{M}^{+}\right.$, 7), 301 (100).

2-Methyl-6-(trimethylsilyl)benzo[c]fluoren-7-one 7i

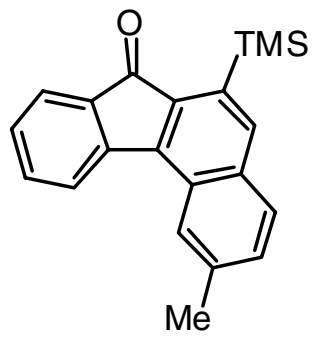

Orange prisms; mp 172-174 ${ }^{\circ} \mathrm{C} ;{ }^{1} \mathrm{H}$ NMR $\left(\mathrm{CDCl}_{3}\right) \delta: 8.16(\mathrm{~s}, 1 \mathrm{H}, \operatorname{Ar} H), 7.97(\mathrm{~d}, J=7.4 \mathrm{~Hz}$, $1 \mathrm{H}, \operatorname{Ar} H), 7.85(\mathrm{~s}, 1 \mathrm{H}, \operatorname{Ar} H), 7.73(\mathrm{~d}, J=7.7 \mathrm{~Hz}, 1 \mathrm{H}, \operatorname{Ar} H), 7.60(\mathrm{~d}, J=7.2 \mathrm{~Hz}, 1 \mathrm{H}, \operatorname{Ar} H)$, 7.47 (t, $J=7.6 \mathrm{~Hz}, 1 \mathrm{H}, \operatorname{Ar} H), 7.36(\mathrm{~d}, J=6.7 \mathrm{~Hz}, 1 \mathrm{H}, \operatorname{Ar} H), 7.25(\mathrm{t}, J=7.4 \mathrm{~Hz}, 1 \mathrm{H}, \operatorname{Ar} H$ ), $2.55\left(\mathrm{~s}, 3 \mathrm{H}, \mathrm{CH}_{3}\right), 0.44\left(\mathrm{~s}, 9 \mathrm{H}, \mathrm{Si}\left(\mathrm{CH}_{3}\right)_{3}\right) ;{ }^{13} \mathrm{C}$ NMR/DEPT $\left(\mathrm{CDCl}_{3}\right) \delta: 195.9(\mathrm{CO}), 145.1$ (C), $142.3(C), 137.9(C), 136.8(\mathrm{CH}), 135.9(C), 135.1(C), 134.2(C \mathrm{H}), 134.1(C), 133.8$ $(C), 130.5(\mathrm{CH}), 129.5(\mathrm{CH}), 129.3(\mathrm{C}), 128.3(\mathrm{CH}), 123.5(\mathrm{CH}), 123.4(\mathrm{CH}), 123.1(\mathrm{CH})$, $22.2\left(\mathrm{CH}_{3}\right),-0.8\left(\mathrm{Si}\left(\mathrm{CH}_{3}\right)_{3}\right)$; $\mathrm{MS}(70 \mathrm{eV}) \mathrm{m} / z(\%): 316\left(\mathrm{M}^{+}, 7\right), 302(30), 301(100)$.

2-Methoxy-6-(trimethylsilyl)benzo[c]fluoren-7-one 7.j<smiles>COc1ccc2cc(C(C)(C)C)c3c(c2c1)-c1ccccc1C3=O</smiles>

Orange prisms; mp 193-194 ${ }^{\circ} \mathrm{C} ;{ }^{1} \mathrm{H}$ NMR $\left(\mathrm{CDCl}_{3}\right) \delta: 7.94(\mathrm{~d}, J=7.9 \mathrm{~Hz}, 1 \mathrm{H}, \mathrm{ArH}), 7.88(\mathrm{~s}$, $1 \mathrm{H}, \operatorname{Ar} H), 7.80(\mathrm{~d}, J=9.1 \mathrm{~Hz}, 1 \mathrm{H}, \operatorname{Ar} H), 7.71(\mathrm{~d}, J=2.3 \mathrm{~Hz}, 1 \mathrm{H}, \operatorname{Ar} H), 7.68-7.63(\mathrm{~m}, 1 \mathrm{H}$, $\mathrm{ArH}), 7.53(\mathrm{td}, J=7.6,1.3 \mathrm{~Hz}, 1 \mathrm{H}, \mathrm{Ar} H), 7.34-7.24(\mathrm{~m}, 2 \mathrm{H}, \mathrm{ArH}), 4.03\left(\mathrm{~s}, 3 \mathrm{H}, \mathrm{OCH}_{3}\right), 0.43$ (s, 9H, $\left.\mathrm{Si}\left(\mathrm{CH}_{3}\right)_{3}\right) ;{ }^{13} \mathrm{C}$ NMR/DEPT $\left(\mathrm{CDCl}_{3}\right) \delta$ : $195.9(\mathrm{CO}), 159.2(\mathrm{C}), 145.2(\mathrm{C}), 141.5(\mathrm{C})$, $136.7(\mathrm{CH}), 136.4(C), 134.2(\mathrm{CH}), 134.1(C), 132.5(C), 131.3(C), 131.1(\mathrm{CH}), 130.2(C)$, $128.2(\mathrm{CH}), 123.7(\mathrm{CH}), 122.6(\mathrm{CH}), 120.8(\mathrm{CH}), 102.7(\mathrm{CH}), 55.4\left(\mathrm{CH}_{3}\right),-0.9\left(\mathrm{Si}\left(\mathrm{CH}_{3}\right)_{3}\right)$; MS (70 eV) m/z (\%): $332\left(\mathrm{M}^{+}, 12\right), 317$ (100), 274 (33).

2-t-Butyl-6-(trimethylsilyl)benzo[c]fluoren-7-one 7k<smiles>Cc1cc2ccc(C(C)(C)C)cc2c2c1C(=O)c1ccccc1-2</smiles> 
Orange prisms; mp $183-184{ }^{\circ} \mathrm{C} ;{ }^{1} \mathrm{H}$ NMR $\left(\mathrm{CDCl}_{3}\right) \delta: 8.43(\mathrm{~s}, 1 \mathrm{H}, \mathrm{ArH}), 8.00(\mathrm{~d}, J=7.5 \mathrm{~Hz}$, 1H, ArH), 7.90 (s, 1H, ArH), 7.84 (d, J= 8.5 Hz, 1H, ArH), 7.71-7.64 (m, 2H, ArH), 7.54 $(\mathrm{t}, J=7.5 \mathrm{~Hz}, 1 \mathrm{H}, \mathrm{ArH}), 7.29(\mathrm{~d}, J=7.5 \mathrm{~Hz}, 1 \mathrm{H}, \mathrm{ArH}), 1.49\left(\mathrm{~s}, 9 \mathrm{H}, \mathrm{C}\left(\mathrm{CH}_{3}\right)_{3}\right), 0.43$ (s, 9H, $\left.\mathrm{Si}\left(\mathrm{CH}_{3}\right)_{3}\right) ;{ }^{13} \mathrm{C} \mathrm{NMR/DEPT}\left(\mathrm{CDCl}_{3}\right) \delta: 196.4(\mathrm{CO}), 151.4(\mathrm{C}), 145.7(C), 143.5(\mathrm{C}), 136.9$ $(C \mathrm{H}), 136.4(C), 135.6(C), 134.7(C H), 134.7(C), 129.8(C H), 129.6(C), 128.8(C H)$, $128.7(C), 127.8(\mathrm{CH}), 124.1(\mathrm{CH}), 123.6(\mathrm{CH}), 119.7(\mathrm{CH}), 35.7(C), 31.5\left(\mathrm{C}\left(\mathrm{CH}_{3}\right)_{3}\right),-0.4$ $\left(\mathrm{Si}\left(\mathrm{CH}_{3}\right)_{3}\right)$; MS (70 eV) m/z (\%): $358\left(\mathrm{M}^{+}, 11\right), 343(100)$.

Benzo[c]fluoren-7-one 71

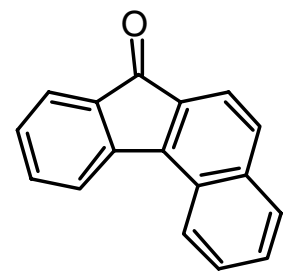

Yellow prisms; mp $158-160^{\circ} \mathrm{C}\left(\right.$ lit $\left.^{1} .161-162^{\circ} \mathrm{C}\right) ;{ }^{1} \mathrm{H}$ NMR $\left(\mathrm{CDCl}_{3}\right) \delta: 8.44(\mathrm{~d}, J=7.5 \mathrm{~Hz}$, 1H, ArH), $7.97(\mathrm{~d}, J=7.5 \mathrm{~Hz}, 1 \mathrm{H}, \operatorname{Ar} H), 7.83$ (d, J=7.2 Hz, 1H, ArH), 7.73 (d, J= 7.8 Hz, $1 \mathrm{H}, \operatorname{Ar} H), 7.68(\mathrm{~d}, J=7.8 \mathrm{~Hz}, 1 \mathrm{H}, \operatorname{ArH}), 7.64(\mathrm{t}, J=7.2 \mathrm{~Hz}, 1 \mathrm{H}, \operatorname{ArH}), 7.57-7.51(\mathrm{~m}, 1 \mathrm{H}$, $\operatorname{ArH}), 7.47(\mathrm{t}, J=7.5 \mathrm{~Hz}, 1 \mathrm{H}, \operatorname{Ar} H), 7.28-7.19(\mathrm{~m}, 2 \mathrm{H}, \mathrm{Ar} H) ;{ }^{13} \mathrm{C} \mathrm{NMR} / \mathrm{DEPT}\left(\mathrm{CDCl}_{3}\right) \delta$ : $194.4(C \mathrm{O}), 144.9(C), 142.8(C), 137.9(C), 134.3(C), 134.4(C \mathrm{H}), 131.7(C), 129.9(C \mathrm{H})$, $129.6(\mathrm{CH}), 128.7(C), 128.6(C \mathrm{H}), 128.2(\mathrm{CH}), 127.7(\mathrm{CH}), 124.7(C \mathrm{H}), 124.0(\mathrm{CH})$, $123.3(\mathrm{CH}), 119.8(\mathrm{CH})$; MS (70 eV) m/z (\%): $230\left(\mathrm{M}^{+}, 100\right), 202$ (38), 201 (22), 200 (22); HRMS $\left(\mathrm{C}_{17} \mathrm{H}_{10} \mathrm{O}\right)$ : calc. 230.0731; found, 230.0727.

4-Methoxy-2-methyl-6-(trimethylsilyl)benzo[c]fluoren-7-one $\mathbf{7 m}$

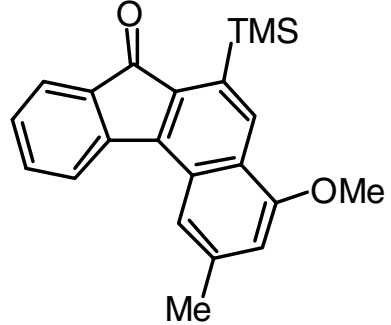

Orange prisms; mp $195{ }^{\circ} \mathrm{C}$ (dec.); ${ }^{1} \mathrm{H}$ NMR $\left(\mathrm{CDCl}_{3}\right) \delta: 8.00(\mathrm{~d}, J=7.9 \mathrm{~Hz}, 1 \mathrm{H}, \mathrm{Ar} H), 7.81$ $(\mathrm{s}, 1 \mathrm{H}, \operatorname{Ar} H), 7.62(\mathrm{~d}, J=7.4 \mathrm{~Hz}, 1 \mathrm{H}, \operatorname{Ar} H), 7.49$ (td, J= 7.4, $1.4 \mathrm{~Hz}, 1 \mathrm{H}, \operatorname{Ar} H), 7.29-7.24$ (m, 2H, ArH), 6.73 (s, 1H, $\mathrm{ArH}), 4.01\left(\mathrm{~s}, 3 \mathrm{H}, \mathrm{OCH}_{3}\right), 2.57$ (s, 3H, CH $), 0.44(\mathrm{~s}, 9 \mathrm{H}$, $\left.\mathrm{Si}\left(\mathrm{CH}_{3}\right)_{3}\right) ;{ }^{13} \mathrm{C}$ NMR/DEPT $\left(\mathrm{CDCl}_{3}\right) \delta: 196.1(\mathrm{CO}), 156.0(\mathrm{C}), 145.4(C), 141.7(\mathrm{C}), 138.5$ (C), 134.2 (CH), 134.1 (C), $132.9(C), 130.4(C), 130.3(C H), 128.2(C H), 127.2(C), 123.5$ $(C \mathrm{H}), 123.3(\mathrm{CH}), 123.2(C), 115.7(\mathrm{CH}), 108.4(\mathrm{CH}), 55.6\left(\mathrm{CH}_{3}\right), 22.8\left(\mathrm{CH}_{3}\right),-0.8$ $\left(\mathrm{Si}\left(\mathrm{CH}_{3}\right)_{3}\right)$; MS (70 eV) m/z (\%): $346\left(\mathrm{M}^{+}, 34\right), 331$ (100), $288(60)$. HRMS $\left(\mathrm{C}_{22} \mathrm{H}_{22} \mathrm{O}_{2} \mathrm{Si}\right)$ : calc. 346.1389; found, 346.1385.

\footnotetext{
${ }^{1}$ Incomplete description, see: Harvey, R. G.; Abu-shqasra, E.; Yang, C. J. Org. Chem. 1992, 57, 6313-6317
} 
2,4-Dimethoxy-6-(trimethylsilyl)benzo[c]fluoren-7-one 7n<smiles></smiles>

Orange prisms; mp 168-170 ${ }^{\circ} \mathrm{C} ;{ }^{1} \mathrm{H}$ NMR $\left(\mathrm{CDCl}_{3}\right) \delta: 8.44(\mathrm{~s}, 1 \mathrm{H}, \mathrm{Ar} H), 7.98(\mathrm{~d}, J=7.7 \mathrm{~Hz}$, $1 \mathrm{H}, \operatorname{Ar} H), 7.73(\mathrm{~d}, J=7.2 \mathrm{~Hz}, 1 \mathrm{H}, \operatorname{Ar} H), 7.59$ (dd, $J=7.7,1.5 \mathrm{~Hz}, 1 \mathrm{H}, \operatorname{Ar} H), 7.37-7.34(\mathrm{~m}$, $2 \mathrm{H}, \operatorname{Ar} H), 6.68(\mathrm{~d}, J=1.5 \mathrm{~Hz}, 1 \mathrm{H}, \mathrm{ArH}), 4.10\left(\mathrm{~s}, 3 \mathrm{H}, \mathrm{OCH}_{3}\right), 4.08\left(\mathrm{~s}, 3 \mathrm{H}, \mathrm{OCH}_{3}\right), 0.51$ (s, 9H, $\left.\mathrm{Si}\left(\mathrm{CH}_{3}\right)_{3}\right) ;{ }^{13} \mathrm{C}$ NMR/DEPT $\left(\mathrm{CDCl}_{3}\right) \delta: 198.2(\mathrm{CO}), 159.9(\mathrm{C}), 157.2(\mathrm{C}), 145.4(\mathrm{C})$, $140.9(C), 137.0(C), 134.1(C H), 134.0(C), 131.4(C), 130.7(C), 130.4(C H), 128.0(C H)$, $125.2(C), 123.6(\mathrm{CH}), 122.6(\mathrm{CH}), 99.6(\mathrm{CH}), 94.7(\mathrm{CH}), 55.8\left(\mathrm{CH}_{3}\right), 55.5\left(\mathrm{CH}_{3}\right),-0.7$ $\left(\mathrm{Si}\left(\mathrm{CH}_{3}\right)_{3}\right) ; \mathrm{MS}(70 \mathrm{eV}) \mathrm{m} / z(\%): 362\left(\mathrm{M}^{+}, 17\right), 348$ (29), 347 (100), 332 (11); HRMS $\left(\mathrm{C}_{22} \mathrm{H}_{22} \mathrm{O}_{3} \mathrm{Si}\right)$ : calc. 362.1338; found, 362.1341 .

\section{2,4-Dimethyl-6-(trimethylsilyl)benzo[c]fluoren-7-one 7o}

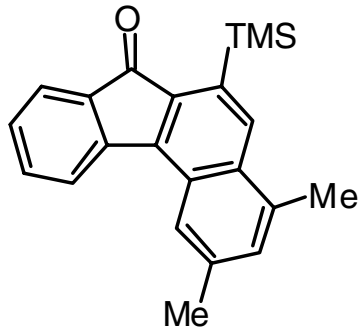

Orange prisms; mp 199-201 ${ }^{\circ} \mathrm{C} ;{ }^{1} \mathrm{H}$ NMR $\left(\mathrm{CDCl}_{3}\right) \delta$ : 8.09-8.02 (m, 2H, $\left.\mathrm{Ar} H\right), 7.96(\mathrm{~d}, J=$ $7.5 \mathrm{~Hz}, 1 \mathrm{H}, \operatorname{Ar} H), 7.59(\mathrm{~d}, J=7.4 \mathrm{~Hz}, 1 \mathrm{H}, \mathrm{ArH}), 7.45(\mathrm{td}, J=7.5,1.2 \mathrm{~Hz}, 1 \mathrm{H}, \operatorname{Ar} H), 7.28-$ $7.20(\mathrm{~m}, 2 \mathrm{H}, \mathrm{ArH}), 2.65\left(\mathrm{~s}, 3 \mathrm{H}, \mathrm{CH}_{3}\right), 2.50\left(\mathrm{~s}, 3 \mathrm{H}, \mathrm{CH}_{3}\right), 0.45\left(\mathrm{~s}, 9 \mathrm{H}, \mathrm{Si}\left(\mathrm{CH}_{3}\right)_{3}\right) ;{ }^{13} \mathrm{C}$ NMR/DEPT $\left(\mathrm{CDCl}_{3}\right)$ \&: $196.0(C O), 145.3(C), 142.5(C), 137.4(C), 135.7(C), 135.6(C)$, $134.2(2 \times C), 134.1(\mathrm{CH}), 133.3(C), 132.5(\mathrm{CH}), 131.5(C), 129.7(\mathrm{CH}), 128.2(\mathrm{CH}), 123.5$ $(\mathrm{CH}), 123.3(\mathrm{CH}), 121.7(\mathrm{CH}), 22.1\left(\mathrm{CH}_{3}\right), 19.9\left(\mathrm{CH}_{3}\right),-0.8\left(\mathrm{Si}\left(\mathrm{CH}_{3}\right)_{3}\right) ; \mathrm{MS}(70 \mathrm{eV}) \mathrm{m} / \mathrm{z}$ (\%): $330\left(\mathrm{M}^{+}, 8\right), 315(100), 285(14)$.

8-(Trimethylsilyl)dibenzo[b,g]fluoren-7-one 16'a

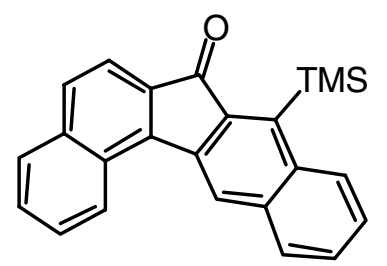

Yellow crystals; mp 160-162 ${ }^{\circ} \mathrm{C} ;{ }^{1} \mathrm{H}$ NMR $\left(\mathrm{CDCl}_{3}\right) \delta: 8.54(\mathrm{~d}, J=8.2 \mathrm{~Hz}, 1 \mathrm{H}, \mathrm{Ar} H), 8.35$ (d, $J=8.2 \mathrm{~Hz}, 1 \mathrm{H}, \operatorname{Ar} H), 8.20$ (s, $1 \mathrm{H}, \operatorname{Ar} H), 7.80-7.76(\mathrm{~m}, 2 \mathrm{H}, \operatorname{Ar} H), 7.68-7.38(\mathrm{~m}, 6 \mathrm{H}$, $\mathrm{ArH}), 0.63$ (s, 9H, $\left.\mathrm{Si}\left(\mathrm{CH}_{3}\right)_{3}\right) ;{ }^{13} \mathrm{C}$ NMR/DEPT $\left(\mathrm{CDCl}_{3}\right) \delta: 194.5(\mathrm{CO}), 143.7(\mathrm{C}), 142.1$ 
(C), $139.0(C), 137.7(C), 137.6(C), 135.6(C), 133.9(C), 130.9(\mathrm{CH}), 129.9(C \mathrm{H}), 129.8$ $(\mathrm{CH}), 129.7(\mathrm{CH}), 128.8(\mathrm{C}), 128.2(\mathrm{CH}), 128.0(\mathrm{CH}), 127.7(\mathrm{CH}), 126.3(\mathrm{CH}), 125.0$ $(\mathrm{CH}), 124.1(\mathrm{C}), 124.1(\mathrm{CH}), 119.5(\mathrm{CH}), 3.0\left(\mathrm{Si}\left(\mathrm{CH}_{3}\right)_{3}\right)$; MS $(70 \mathrm{eV}) \mathrm{m} / \mathrm{z}(\%): 352\left(\mathrm{M}^{+}\right.$, 9), 338 (31), 337 (100), 307 (23); HRMS $\left(\mathrm{C}_{24} \mathrm{H}_{20} \mathrm{OSi}\right)$ : calc. 352.1283; found, 352.1291.

\section{Dibenzo[b,g]fluoren-7-one 16a}

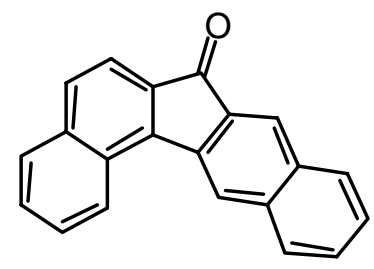

Yellow prisms; $\mathrm{mp} 203-205{ }^{\circ} \mathrm{C} ;{ }^{1} \mathrm{H}$ NMR $\left(\mathrm{CDCl}_{3}\right) \delta$ : $8.60(\mathrm{~d}, J=7.3 \mathrm{~Hz}, 1 \mathrm{H}, \mathrm{Ar} H), 8.22$ (s, $1 \mathrm{H}, \mathrm{Ar} H), 8.07(\mathrm{~s}, 1 \mathrm{H}, \mathrm{Ar} H), 7.88-7.42(\mathrm{~m}, 9 \mathrm{H}, \mathrm{ArH}) ;{ }^{13} \mathrm{C}$ NMR/DEPT $\left(\mathrm{CDCl}_{3}\right) \delta: 192.8$ (CO), $142.9(C), 138.6(C), 137.7(C), 136.6(C), 134.2(C), 133.0(C), 132.8(C), 130.3$ $(\mathrm{CH}), 129.9(\mathrm{CH}), 129.5(\mathrm{CH}), 129.0(\mathrm{CH}), 128.9(\mathrm{C}), 128.7(\mathrm{CH}), 128.3(\mathrm{CH}), 127.6(\mathrm{CH})$, 127.0 $(\mathrm{CH}), 125.0(\mathrm{CH}), 124.8(\mathrm{CH}), 122.4(\mathrm{CH}), 119.6(\mathrm{CH}) ; \mathrm{MS}(70 \mathrm{eV}) \mathrm{m} / \mathrm{z}(\%): 280$ $\left(\mathrm{M}^{+}, 100\right), 252$ (38), 125 (28); HRMS $\left(\mathrm{C}_{21} \mathrm{H}_{12} \mathrm{O}\right)$ : calc. 280.0888; found, 280.0883.

10-Methoxy-8-(trimethylsilyl)dibenzo[b,g]fluoren-7-one 16'b

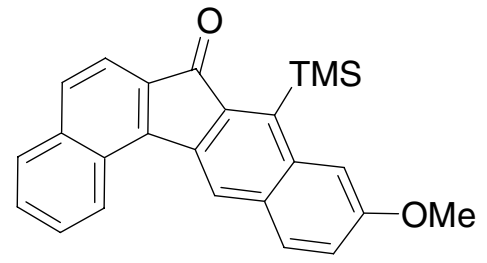

Yellow crystals; mp $243-245{ }^{\circ} \mathrm{C} ;{ }^{1} \mathrm{H}$ NMR $\left(\mathrm{CDCl}_{3}\right) \delta: 8.68(\mathrm{~d}, J=8.2 \mathrm{~Hz}, 1 \mathrm{H}, \mathrm{Ar} H), 8.31$ $(\mathrm{s}, 1 \mathrm{H}, \operatorname{Ar} H), 7.90(\mathrm{~d}, J=8.2 \mathrm{~Hz}, 1 \mathrm{H}, \operatorname{Ar} H), 7.80(\mathrm{~d}, J=2.4 \mathrm{~Hz}, 1 \mathrm{H}, \operatorname{Ar} H), 7.78-7.23(\mathrm{~m}$, $3 \mathrm{H}, \operatorname{Ar} H), 7.68(\mathrm{td}, J=6.9,1.3 \mathrm{~Hz}, 1 \mathrm{H}, \operatorname{Ar} H), 7.61(\mathrm{td}, J=6.9,1.3 \mathrm{~Hz}, 1 \mathrm{H}, \operatorname{Ar} H), 7.19(\mathrm{dd}$, $J=8.8,2.5 \mathrm{~Hz}, 1 \mathrm{H}, \mathrm{Ar} H), 3.94\left(\mathrm{~s}, 3 \mathrm{H}, \mathrm{OCH}_{3}\right), 0.63\left(\mathrm{~s}, 9 \mathrm{H}, \mathrm{Si}\left(\mathrm{CH}_{3}\right)_{3}\right) ;{ }^{13} \mathrm{C}$ NMR/DEPT $\left(\mathrm{CDCl}_{3}\right)$ 8: $190.7(\mathrm{CO}), 158.3(\mathrm{C}), 142.8(\mathrm{C}), 142.1(\mathrm{C}), 141.0(\mathrm{C}), 139.1(\mathrm{C}), 138.0(\mathrm{C})$, $137.5(C), 133.5(C), 131.0(C H), 130.6(C), 129.8(C H), 128.8(C), 128.3(C H), 127.7$ $(\mathrm{CH}), 125.2(\mathrm{CH}), 124.1(\mathrm{CH}), 119.9(\mathrm{CH}), 119.8(\mathrm{CH}), 119.7(\mathrm{CH}), 111.1(\mathrm{CH}), 55.5$ $\left(\mathrm{CH}_{3}\right), 3.1\left(\mathrm{Si}\left(\mathrm{CH}_{3}\right)_{3}\right) ; \mathrm{MS}(70 \mathrm{eV}) \mathrm{m} / z(\%): 382\left(\mathrm{M}^{+}, 21\right), 367$ (100), 324 (28).

10-Methoxydibenzo[b,g]fluoren-7-one $\mathbf{1 6 b}$

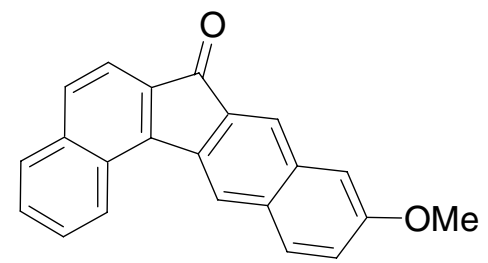

Yellow crystals; mp 222-225 ${ }^{\circ} \mathrm{C} ;{ }^{1} \mathrm{H}$ NMR $\left(\mathrm{CDCl}_{3}\right) \delta: 8.59(\mathrm{~d}, J=8.5 \mathrm{~Hz}, 1 \mathrm{H}, \mathrm{Ar} H), 8.17$ $(\mathrm{s}, 1 \mathrm{H}, \operatorname{Ar} H), 7.99(\mathrm{~s}, 1 \mathrm{H}, \operatorname{Ar} H), 7.87(\mathrm{~d}, J=8.0 \mathrm{~Hz}, 1 \mathrm{H}, \operatorname{Ar} H), 7.76-7.71(\mathrm{~m}, 3 \mathrm{H}, \operatorname{Ar} H)$, $7.65(\mathrm{td}, J=6.8,1.4 \mathrm{~Hz}, 1 \mathrm{H}, \operatorname{Ar} H), 7.59(\mathrm{td}, J=6.8,1.4 \mathrm{~Hz}, 1 \mathrm{H}, \operatorname{Ar} H), 7.19-7.14(\mathrm{~m}, 2 \mathrm{H}$, 
$\mathrm{ArH}), 3.92\left(\mathrm{~s}, 3 \mathrm{H}, \mathrm{OCH}_{3}\right) ;{ }^{13} \mathrm{C} \mathrm{NMR} / \mathrm{DEPT}\left(\mathrm{CDCl}_{3}\right) \delta: 193.5(\mathrm{CO}), 158.7(C), 143.7(C)$, $138.0(C), 136.9(C), 134.4(C), 133.9(C), 133.8(C), 130.4(2 \times C H), 129.62(C H), 129.6$ $(C), 128.4(C H), 127.6(C H), 127.5(C), 125.2(C H), 124.0(C H), 122.5(C H), 120.7(C H)$, $119.8(C \mathrm{H}), 109.4(\mathrm{CH}), 55.4\left(\mathrm{CH}_{3}\right)$; MS (70 eV) m/z (\%): $310\left(\mathrm{M}^{+}, 100\right), 267(58), 239$ (44).

4-Methoxy-7H-benzo[c]fluorene 23. a) Desilylation of 7c: Tetrabutylammonium fluoride (0.3 mL, $0.3 \mathrm{mmol}, 1 \mathrm{M}$ in THF) was added to a solution of $7 \mathrm{c}(65 \mathrm{mg}, 0.2 \mathrm{mmol})$ in commercial grade toluene $(8 \mathrm{~mL})$, and the resulting mixture was heated at $90{ }^{\circ} \mathrm{C}$ for 75 min. After solvent concentration, the residue was dissolved in $\mathrm{CH}_{2} \mathrm{Cl}_{2}$, washed with aqueous $\mathrm{HCl}(5 \%)$ and saturated brine, dried over $\mathrm{Na}_{2} \mathrm{SO}_{4}$, and concentrated to dryness giving the desilylated ketone 7c' (51 mg, 100\%) as red crystals, m.p. 200-201 ${ }^{\circ} \mathrm{C}$.

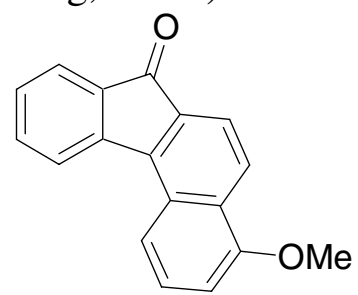

${ }^{1} \mathrm{H}$ NMR $\left(\mathrm{CDCl}_{3}\right) \delta: 8.29(\mathrm{dd}, J=8.5,1.0 \mathrm{~Hz}, 1 \mathrm{H}, \mathrm{ArH}), 8.09-7.99$ (m, 2H, ArH), $7.73(\mathrm{~d}$, $J=8.5 \mathrm{~Hz}, 1 \mathrm{H}, \operatorname{Ar} H), 7.71-7.66(\mathrm{~m}, 1 \mathrm{H}, \operatorname{ArH}), 7.54(\mathrm{dd}, J=8.5,7.9 \mathrm{~Hz}, 1 \mathrm{H}, \operatorname{ArH}), 7.52(\mathrm{td}$, $J=7.5,1.3 \mathrm{~Hz}, 1 \mathrm{H}, \operatorname{ArH}), 7.30(\mathrm{~d}, J=7.5 \mathrm{~Hz}, 1 \mathrm{H}, \operatorname{Ar} H), 6.92(\mathrm{~d}, J=7.5 \mathrm{~Hz}, 1 \mathrm{H}, \operatorname{Ar} H), 4.03$ $\left(\mathrm{s}, 3 \mathrm{H}, \mathrm{OCH}_{3}\right) ;{ }^{13} \mathrm{C} \mathrm{NMR} / \mathrm{DEPT}\left(\mathrm{CDCl}_{3}\right) \delta: 194.6(\mathrm{CO}), 156.0(C), 145.2(C), 142.1(C)$, $134.4(C+C H), 132.1(C), 130.0(C), 129.6(C), 128.4(C H), 127.9(C H), 123.9(C H)$, $123.8(\mathrm{CH}), 123.3(\mathrm{CH}), 118.9(\mathrm{CH}), 116.8(\mathrm{CH}), 105.9(\mathrm{CH}), 55.5\left(\mathrm{CH}_{3}\right) ; \mathrm{MS}(70 \mathrm{eV}) \mathrm{m} / z$ (\%): $260\left(\mathrm{M}^{+}, 100\right), 217$ (59), 189 (49).

b) Hydrogenation: To a solution of 7c' $(51 \mathrm{mg}, 0.2 \mathrm{mmol})$ in THF (5 mL) was succesively added $12 \mathrm{~mL}$ of aqueous $\mathrm{HCl}(5 \%)$ and $50 \mathrm{mg}$ of $10 \% \mathrm{Pd} / \mathrm{C}$. The heterogeneous mixture, heated at $55{ }^{\circ} \mathrm{C}$, was stirred under $\mathrm{H}_{2}$ (balloon) for $4 \mathrm{~h}$. The mixture was rapidly filtered, extracted with EtOAc and washed with saturated brine. The organic layer was dried over anhydrous $\mathrm{Na}_{2} \mathrm{SO}_{4}$ and concentrated giving 4-methoxy-7H-benzo[c]fluorene 23 (49mg, $100 \%$ ) as pale brown crystals, m.p. $112-114^{\circ} \mathrm{C}$.

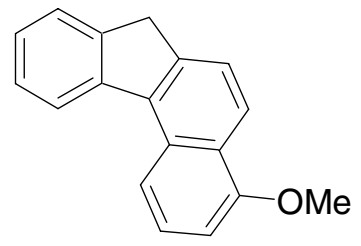

${ }^{1} \mathrm{H}$ NMR $\left(\mathrm{CDCl}_{3}\right) \delta: 8.42-8.27(\mathrm{~m}, 3 \mathrm{H}, \mathrm{ArH}), 7.69(\mathrm{~d}, J=8.5 \mathrm{~Hz}, 1 \mathrm{H}, \mathrm{ArH}), 7.64(\mathrm{~d}, J=7.5$ $\mathrm{Hz}, 1 \mathrm{H}, \operatorname{ArH}), 7.57$ (t, J=7.6 Hz, 1H, ArH), 7.49 (t, J=7.6 Hz, 1H, ArH), 7.35 (td, $J=7.5$, $0.9 \mathrm{~Hz}, 1 \mathrm{H}, \mathrm{ArH}), 6.90(\mathrm{~d}, J=7.5 \mathrm{~Hz}, 1 \mathrm{H}, \mathrm{ArH}), 4.05\left(\mathrm{~s}, 3 \mathrm{H}, \mathrm{OCH}_{3}\right), 4.03\left(\mathrm{~s}, 2 \mathrm{H}, \mathrm{CH}_{2}\right) ;{ }^{13} \mathrm{C}$ NMR/DEPT $\left(\mathrm{CDCl}_{3}\right) \delta: 156.1(C), 144.1(C), 142.9(C), 142.8(C), 135.8(C), 130.6(C)$, $126.8(\mathrm{CH}), 126.6(\mathrm{CH}), 125.6(\mathrm{CH}), 125.3(C), 124.8(\mathrm{CH}), 122.9(\mathrm{CH}), 122.4(C \mathrm{H})$, $121.3(\mathrm{CH}), 116.2(\mathrm{CH}), 103.3(\mathrm{CH}), 55.5\left(\mathrm{CH}_{3}\right), 37.6\left(\mathrm{CH}_{2}\right) ; \mathrm{MS}(70 \mathrm{eV}) \mathrm{m} / \mathrm{z}(\%): 246$ $\left(\mathrm{M}^{+}, 100\right), 231$ (34), 202 (77), 101 (31). 
7H-Benzo[c]fluoren-4-ol 24. To a solution of 23 (48 mg, $0.2 \mathrm{mmol})$ in $\mathrm{AcOH}(3 \mathrm{~mL})$ was added $3 \mathrm{~mL}$ of aqueous $\mathrm{HBr}(48 \%)$, and the resulting mixture was heated under argon at $130{ }^{\circ} \mathrm{C}$ for $4 \mathrm{~h}$. The mixture was extracted with EtOAc and washed with saturated $\mathrm{Na}_{2} \mathrm{CO}_{3}$, dried over anhydrous $\mathrm{Na}_{2} \mathrm{SO}_{4}$ and concentrated. The residue was purified by column chromatography on silica gel using EtOAc/hexanes 1:9 as eluent giving $\mathbf{2 4}(39 \mathrm{mg}, 87 \%)$ as white crystals, mp $175-176^{\circ} \mathrm{C}$.

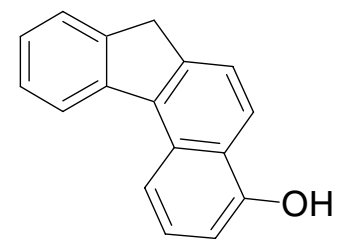

${ }^{1} \mathrm{H}$ NMR $\left(\mathrm{CDCl}_{3}\right) \delta: 8.41-8.34(\mathrm{~m}, 2 \mathrm{H}, \mathrm{ArH}), 8.23(\mathrm{~d}, J=8.6 \mathrm{~Hz}, 1 \mathrm{H}, \mathrm{ArH}), 7.71(\mathrm{~d}, J=8.6$ $\mathrm{Hz}, 1 \mathrm{H}, \operatorname{Ar} H), 7.65(\mathrm{~d}, J=7.5 \mathrm{~Hz}, 1 \mathrm{H}, \mathrm{ArH}), 7.54-7.44(\mathrm{~m}, 2 \mathrm{H}, \mathrm{ArH}), 7.36$ (t, J= 7.5, Hz, $1 \mathrm{H}, \operatorname{ArH}), 6.88(\mathrm{~d}, J=7.6 \mathrm{~Hz}, 1 \mathrm{H}, \mathrm{ArH}), 5.32$ (broad s, $1 \mathrm{H}, \mathrm{OH}), 4.03\left(\mathrm{~s}, 2 \mathrm{H}, \mathrm{CH}_{2}\right) ;{ }^{13} \mathrm{C}$ NMR/DEPT $\left(\mathrm{CDCl}_{3}\right)$ \&: $152.1(C), 144.1(C), 143.0(C), 142.8(C), 135.9(C), 130.9(C)$, $126.9(\mathrm{CH}), 126.5(\mathrm{CH}), 125.7(\mathrm{CH}), 124.8(\mathrm{CH}), 124.1(C), 122.9(C \mathrm{H}), 122.6(C \mathrm{H})$, $121.0(\mathrm{CH}), 116.7(\mathrm{CH}), 108.1(\mathrm{CH}), 37.7\left(\mathrm{CH}_{2}\right) ; \mathrm{MS}(70 \mathrm{eV}) \mathrm{m} / z(\%): 232\left(\mathrm{M}^{+}, 100\right), 231$ (32), 202 (38). 